\title{
Bifurcations from an Invariant Circle for Two-Parameter Families of Maps of the Plane:sp A Computer-Assisted Study
}

\author{
D. G. Aronson ${ }^{1}$, M. A. Chory ${ }^{2}$, G. R. Hall ${ }^{1}$, and R. P. McGehee ${ }^{1}$ \\ 1 School of Mathematics, University of Minnesota, Minneapolis, MN 55455, USA \\ 2 The Analytic Sciences Corporation, Six Jacob Way, Reading, MA 01867, USA
}

\begin{abstract}
We consider a two-parameter family of maps of the plane to itself. Each map has a fixed point in the first quadrant and is a diffeomorphism in a neighborhood of this point. For certain parameter values there is a Hopf bifurcation to an invariant circle, which is smooth for parameter values in a neighborhood of the bifurcation point. However, computer simulations show that the corresponding invariant set fails to be even topologically a circle for parameter values far from the bifurcation point. This paper is an attempt to elucidate some of the mechanisms involved in this loss of smoothness and alteration of topological type.
\end{abstract}

\section{Stochastic Behavior in Deterministic Systems}

In 1899 Poincaré remarked on the complexity of the behavior of dynamical systems having what he called a "homoclinic point" [21]. The depth of this complexity was illustrated by Smale, who showed that stochastic behavior is associated with a non-degenerate homoclinic point [27]. More precisely, Smale constructed an invariant Cantor set on which the dynamical system is equivalent to a Bernoulli shift. Since Bernoulli shifts model such random processes as cointossing experiments, Smale proved the existence of stochastic behavior in certain deterministic systems.

The invariant Cantor sets discussed by Smale are unstable in the sense that nearby orbits tend away from them. That "stable" invariant sets can also behave stochastically has been appreciated only recently. The appropriate notion here is that of an "attracting" invariant set, or an "attractor." Roughly speaking, an attractor is an invariant set with the property that nearby orbits are drawn to it asymptotically.

A class of attractors exhibiting stochastic behavior has been discussed by Williams [30], Ruelle [24], and Bowen [3]. These so-called "expanding attractors" have a certain uniform hyperbolic structure which makes them tractable to 
analysis. Ruelle and Takens coined the term "strange attractor" as a nickname for "expanding attractor" [26].

Mainly as a result of computer simulations, the name "strange attractor" has been applied to objects which are not necessarily expanding attractors. On the other hand, as Ruelle points out in [25], there is as yet no completely satisfactory mathematical definition of strange attractor which is universally accepted. Here we shall use an operational definition which seems to conform to common usage and which is useful when studying dynamical systems on a computer. Specifically, a dynamical system is said to have a strange attractor if there is an orbit which when followed for a long time does not appear to converge to a fixed point, a periodic orbit, or a quasi-periodic orbit. Although this definition is maddeningly vague and subjective, it is often quite clear in practice when a strange attractor has been found.

Stein and Ulam were apparently the first mathematicians to observe strange attractors on a computer [28]. Lorenz later studied a meteorological model with a strange attractor, now known as the "Lorenz attractor" [17]. Although the Lorenz attractor exhibits a certain stochastic behavior, it is not an expanding attractor $[9,11]$. More recently, Hénon found a quadratic map of the plane with a strange attractor [13]. He illustrated what might be regarded as a Cantor set structure in his attractor.

Much of the computer work has involved maps of the plane. As dynamical systems, these maps have two state variables and a discrete time variable. An iteration of the map corresponds to following the system through one unit of time. If the map is invertible, then the system is reversible and can be followed both forward and backward in time. An invertible map of the plane can be suspended to give a flow in a three-dimensional space and hence can be generated by a differential equation in three state variables. Conversely, if an appropriate section exists, then the solutions of an autonomous differential equation in three state variables give rise to an invertible two-dimensional map. Properties of twodimensional discrete systems thus have analogs for three-dimensional differential equations.

Typically, one studies a one parameter family of maps which have several fixed points. The number of fixed points and their stability properties depend on the parameter value. A particularly interesting transistion is the so-called Hopf bifurcation for maps. As the parameter varies, a fixed point loses stability and spawns a smooth invariant circle. In the case of interest here, the invariant circle is an attractor which grows as the parameter continues to change. The analog for differential equations is a periodic orbit which becomes unstable and spawns an attracting invariant torus.

Curry and Yorke studied a parameterized family of maps of the plane [6]. At a certain parameter value, a Hopf bifurcation occurs. As the parameter changes, the attracting invariant circle grows and starts to warp, eventually becoming a strange attractor. The question arises : how can a smooth invariant circle become a strange attractor?

In this paper we report on a computer assisted study of a simple one parameter family of quadratic maps of the plane. Just as in the Curry-Yorke case, a fixed point bifurcates into a smooth invariant circle which somehow transforms itself 
into a strange attractor as the parameter is increased. Our object is to elucidate the mechanism by which this transformation occurs.

We first attempted to pin down the precise parameter value at which the circle loses its integrity. To our surprise, we found that there is no unique parameter value which divides nice behavior from strange behavior. There are parameter intervals for which the attractor appears to be a smooth invariant circle interspersed among parameter intervals for which the attractor appears to be strange. The more precision we used in our investigation, the more interspersed intervals we found. We tried to catalog various types of behavior, but could find no simple patterns.

Inspired by the work of Arnol'd [1], we decided to embed our system in a twoparameter family. As we investigated this two-parameter family, many patterns began to emerge. It became clear how the smooth invariant circle can lose its smoothness. It also became clear how the attractor can cease to be even topologically a circle. Both of these phenomena are related to behavior associated with the "homoclinic" and "heteroclinic" points defined by Poincaré.

Ruelle and Takens have suggested that strange attractors might provide an explanation for the phenomenon of turbulence in classical fluid dynamics [26]. If they are correct, then our work can be regarded as a study of how the transition from a non-turbulent to a turbulent state can occur for parameterized families of maps of the plane. In this context, part of our conclusion can be stated as follows. In one parameter families, the transistion to turbulence is itself a turbulent process.

We consider this paper to be a blend of computer experiment and mathematical theory. Sections 6 and 7 contain theorems relating the nonexistence and existence of homoclinic orbits to uniqueness and nonuniqueness of rotation numbers. Section 8 contains a rigorous discussion relating the loss of smoothness of an invariant circle to the formation of a certain type of heteroclinic orbit. That the hypotheses of Sects. 6-8 are satisfied for the model we study is supported by computer simulation, but is not proved. However, it was the computer studies of the model which suggested the mathematics, not vice versa.

\section{Annular Attractor Blocks}

Henceforth we restrict our attention to two-dimensional discrete dynamical systems, which we write as maps of the plane $F: R^{2} \rightarrow R^{2}$. Iteration of the map corresponds to evolution in time; if the system starts with initial state $x \in R^{2}$, then its state after $n$ units of time is $F^{n}(x)$. An orbit for $F$ is a bi-infinite sequence of points $x_{n}$ such that $x_{n+1}=F\left(x_{n}\right)$ for all $n$. The positive semi-orbit through $x$ is the infinite sequence $\left(x, F(x), F^{2}(x), \ldots\right)$. Unless otherwise noted, we assume that $F$ is one-to-one, at least in the region of interest.

Definition. Let $B$ be a compact subset of the plane. We call $B$ an attractor block if $F(B)$ is a subset of the interior of $B$. Let

$$
A=\bigcap_{n>0} F^{n}(B)
$$




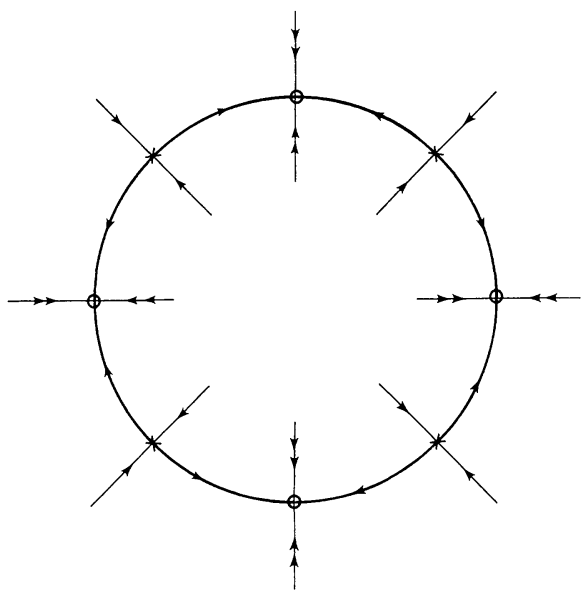

Fig. 2.1. Schematic drawing of an attractor which is a circle in resonance. Saddle points are indicated by $\times$ and sinks by 0

Then $A$ is called an attractor; specifically, $A$ is the attractor associated with attractor block $B$. A subset of $A$ is called a subattractor of $A$ if it is itself an attractor.

An attractor $A$ has two stability properties. First, orbits starting nearby tend to $A$ asymptotically. Second, the attractor block remains an attractor block under small perturbations of the map $F$.

Some authors impose a further condition which $A$ must satisfy to be called an attractor [25]. They require that $A$ should have some recurrence, or indecomposability, property. Conley has proved that an attractor has no subattractor if and only if it satisfies a weak recurrence property called "chain recurrence" [4]. However, he does not impose this condition in the definition of attractor, and we have adopted his definition.

The attractors of primary interest to us are those for which the attractor block $B$ is an annular region in the plane. A case of special interest occurs when $A$ is a circle containing two periodic orbits, one stable and the other unstable (see Fig. 2.1). In this case we refer to $A$ as a "circle in resonance." When resonance occurs, most orbits tend asymptotically to the stable periodic orbit, which is a subattractor within the invariant circle $A$. Simple computer simulations will show only this subattractor and not the whole circle.

By "simple computer simulation" we mean the following. Starting with some initial point $x$, compute $y=F^{n}(x)$, where $n$ is large (at least several thousand). The output is then the positive semi-orbit of $y$. Obviously, the output can depend on the starting point $x$, in which case it may be important to choose several different points and repeat the procedure.

Even in cases when $A$ is not a circle in resonance, simple computer simulation often produces only a portion of $A$. While working with the computer, we found ourselves referring to the portion of $A$ found by the machine as the "visible attractor," while referring to the rest of $A$ as the "invisible attractor." These are not mathematical concepts, only useful labels for computer output. 
It would be nice to be able to equate the visible attractor with a chain recurrent subattractor. However, Conley has shown that there are systems for which none of the attractors is chain recurrent [4]. Although there is always a chain recurrent "quasi-attractor" [4], even this concept is not equivalent to our "visible attractor." There are chain recurrent quasi-attractors which are not "visible" to a computer. Thus we are left without a mathematical definition of the concept of "visible attractor."

The invisible attractor often can be illuminated by a study of the unstable manifolds of the unstable periodic points. To be more precise we must first define these stable and unstable manifolds.

Let $y$ be a periodic point of period $q$, i.e., $F^{q}(y)=y$, and $F^{n}(y) \neq y$ for $0<n<q$. The periodic orbit associated with $y$ is the set

$$
Y=\left\{y, F(y), F^{2}(y), \ldots, F^{q-1}(y)\right\} .
$$

The stable manifold of $Y$ is the set

$$
W^{s}(Y, F)=\left\{x: d\left(F^{n}(x), Y\right) \rightarrow 0 \quad \text { as } \quad n \rightarrow+\infty\right\} .
$$

The unstable manifold of $Y$ is the set

$$
W^{u}(Y, F)=\left\{x: d\left(F^{n}(x), Y\right) \rightarrow 0 \quad \text { as } \quad n \rightarrow-\infty\right\} .
$$

If $z$ is a fixed point, then $z$ is a periodic orbit with period 1 and the above definitions reduce to

$$
\begin{array}{lll}
W^{s}(z, F)=\left\{x: F^{n}(x) \rightarrow z\right. & \text { as } & n \rightarrow+\infty\}, \\
W^{u}(z, F)=\left\{x: F^{n}(x) \rightarrow z\right. & \text { as } & n \rightarrow-\infty\} .
\end{array}
$$

Since $y$ is periodic with period $q$, it is a fixed point for $F^{q}$. It can be shown that

$$
W^{s}(Y, F)=\bigcup_{k=0}^{q-1} W^{s}\left(F^{k}(y), F^{q}\right)
$$

i.e., that the stable manifold of the periodic orbit $Y$ is the union of the stable manifolds of each point of the orbit under the $q^{\text {th }}$ iterate of $F$. The same statement is true for the unstable manifolds.

For the map $F: R^{2} \rightarrow R^{2}$, denote by $D F(x)$ the Jacobian matrix for $F$ evaluated at the point $x$. Let $z$ be a fixed point for $F$ and let $\lambda_{1}$ and $\lambda_{2}$ be the eigenvalues of $D F(z)$. We call $z$ a saddle point if $\left|\lambda_{1}\right|<1$ and $\left|\lambda_{2}\right|>1$. The stable manifold theorem states that $W^{s}(z, F)$ is a curve tangent at $z$ to the eigenspace corresponding to $\lambda_{1}$, while $W^{u}(z, F)$ is a curve tangent at $z$ to the eigenspace corresponding to $\lambda_{2}$ [12]. These curves are as smooth as the map $F$.

Note that these curves do not behave like solution curves of a differential equation. In particular, it is possible for them to cross without coinciding exactly.

Consider again the periodic point $y$ with its periodic orbit $Y$. We call the eigenvalues of the Jacobian matrix $D F^{q}(y)$ the "eigenvalues of the periodic point $y$." Note that the eigenvalues of $y$ are the same as the eigenvalues of $F^{k}(y)$ for any $k$. Hence these eigenvalues are associated with the periodic orbit $Y$ and are called also the "eigenvalues of the periodic orbit $Y$." Note also that these eigenvalues are exactly the eigenvalues associated with $y$ regarded as a fixed point of $F^{q}$. 
We say that $y$ is a periodic saddle if its eigenvalues $\lambda_{1}$ and $\lambda_{2}$ satisfy $\left|\lambda_{1}\right|<1<\left|\lambda_{2}\right|$. The above considerations show that in this case $W^{s}(Y, F)$ is the union of $q$ curves, each passing through one of the points in the orbit. The same is true for $W^{u}(Y, F)$. We say that $y$ is a periodic sink if its eigenvalues satisfy $\left|\lambda_{1}\right|<1$ and $\left|\lambda_{2}\right|<1$. Note that, if $y$ is a periodic sink, then $Y$ is an attractor.

In case the attractor $A$ is an invariant circle in resonance, then it is equal to the closure of the unstable manifold of the periodic saddle, i.e., to the union of this unstable manifold and the orbit of the periodic sink. In this case one can see the invisible attractor by computing the unstable manifold. Even when $A$ is not a circle, much of its structure can be illuminated by computing the unstable manifold of a periodic saddle contained in it.

A useful tool for studying maps of the circle has been the concept of rotation number. The rotation number for points in an annular attractor block can be defined as follows. Introduce polar coordinates $x=(r, \theta)$ on the annulus. Let $\left(r_{n}, \theta_{n}\right)$ be the coordinates of $F^{n}(x)$. We must be careful to point out here that $F$ is taken to be continuous in polar coordinates, so that the $\theta$-coordinate of $F(r, \theta)$ has no jumps. Stated in different terminology, we are considering a lift of $F$ to the covering space of the annulus. Now let

$$
\varrho(x)=\lim _{n \rightarrow \infty} \frac{\theta_{n}}{2 \pi n} .
$$

If this limit exists, we call $\varrho(x)$ the rotation number of the point $x$. If the limit does not exist, then we say that the rotation number of $x$ does not exist.

For homeomorphisms of the circle, the rotation number is independent of the starting point, and we speak of the rotation number of the map. For maps of the annulus, one cannot expect the rotation number to be independent of the starting point, unless $A$ is a circle. In fact, if the rotation number is not unique, then $A$ cannot be a circle. Levinson used this last argument to conclude the existence of a strange separating set in a certain forced nonlinear oscillator by constructing two stable periodic orbits with different rotation numbers inside an annular attractor block [16].

\section{A Model of Delayed Regulation}

In his book, Mathematical Ideas in Biology, John Maynard Smith models the growth of a population whose ability to reproduce in any given generation is governed by the population in the previous generation [18]. Let $N_{n}$ be the population density in the $n^{\text {th }}$ generation and let $a$ be a parameter reflecting the growth rate. Then in its simplest form Maynard Smith's model is

$$
N_{n+1}=a N_{n}\left(1-N_{n-1}\right) .
$$

Note that this is the standard discrete logistic model except that the nonlinear term regulating the population size contains a time delay of one generation. Consequently we shall refer to (3.1) as the "delayed regulation" model. 


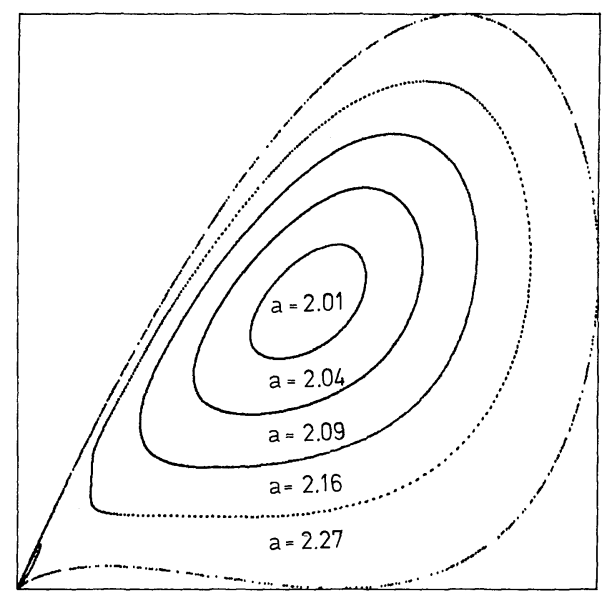

Fig. 3.1. The visible attractor for the delayed logistic map, Eq. (3.2), for various values of the parameter a. Each of the sets shown consists of iterates $1000-2500$ of the point $(0.1,0.2)$

Equation (3.1) can be transformed into a two dimensional system by the introduction of new variables

$$
x_{n}=N_{n-1}, y_{n}=N_{n} .
$$

Then

$$
\begin{aligned}
& x_{n+1}=y_{n} \\
& y_{n+1}=a y_{n}\left(1-x_{n}\right),
\end{aligned}
$$

i.e.,

$$
\left(x_{n+1}, y_{n+1}\right)=F_{a}\left(x_{n}, y_{n}\right),
$$

where $F_{a}$ is the map of the plane defined by

$$
F_{a}(x, y)=(y, a y(1-x))
$$

Note that $F_{a}$ is a one parameter family of maps of the plane, with $a$ as the parameter.

The map $F_{a}$ has a fixed point at

$$
x=y=\frac{a-1}{a},
$$

which is stable for $1<a \leqq 2$. As a passes through the value 2 , this fixed point loses stability and spawns an attracting invariant circle via a Hopf bifurcation. The circle is shown for various parameter values in Fig. 3.1. Note that it grows as the parameter $a$ increases, becoming noticably warped by $a=2.16$. When $a$ reaches 2.27 , the circle has completely broken down, leaving a strange attractor. Various blow-ups of this strange attractor are shown in Fig. 3.2, where a finer and finer structure appears with each successive enlargement. One should note here the similarity to the pictures obtained by Hénon [13]. 


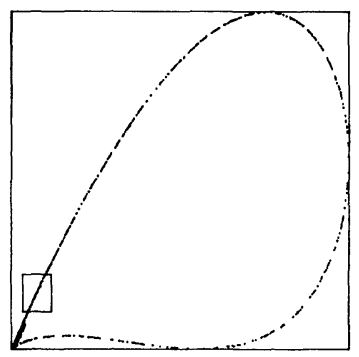

(a)

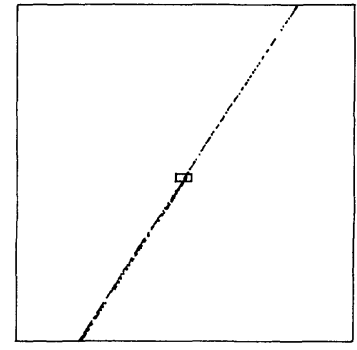

(b)

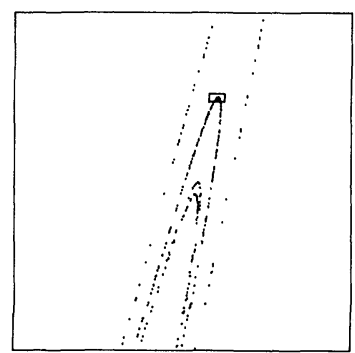

(d)

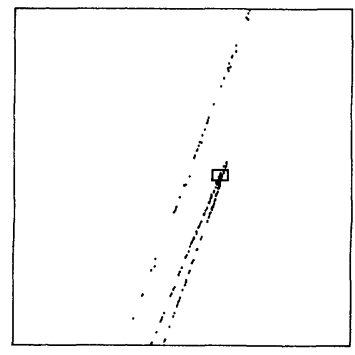

(c)

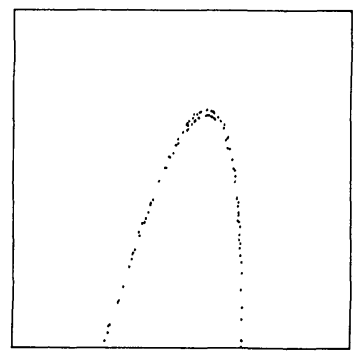

(e)

Fig. 3.2. a The visible attractor for the delayed logistic map for $a=2.27$. Successive enlargements of a portion of the visible attractor. Each picture shows the boxed region from the preceding picture. Scales: a $x \in[0,1], y \in[0,1]$. b $x \in[0.040625,0.1140625], y \in[0.1203125,0.2203125]$. c $x \in[0.07516357$, $0.07895020], y \in[0.1675,0.17046875]$, d $x \in[0.07735272,0.07758346], y \in[0.16891479,0.16903076]$. e $x \in[0.07748395,0.07749910], y \in[0.16899833,0.16900286]$

In addition to the fixed point defined by (3.3), the map $F_{a}$ has another fixed point at the origin. Before discussing the role of this fixed point, we digress briefly to define homoclinic orbits.

Definition. Let $z$ be a fixed point for the map $F$. The orbit $\left\{x_{n}\right\}$ is said to be homoclinic to $z$ if $x_{n} \neq z$ for some $n$ and if

$$
x_{n} \rightarrow z \text { as } n \rightarrow+\infty \text { and as } n \rightarrow-\infty .
$$

A homoclinic point is a point on a homoclinic orbit.

We see that a homoclinic point $x$ is a point in the intersection of the stable and unstable manifolds of $z$. If these manifolds intersect transversely at $x$, then $x$ is 


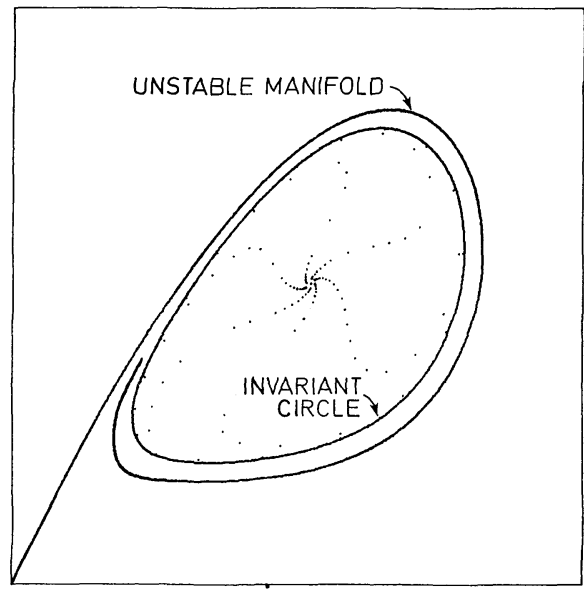

(a)

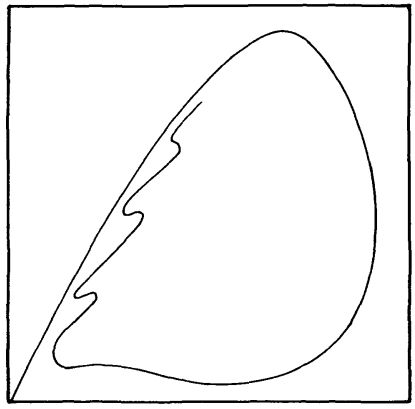

(b)

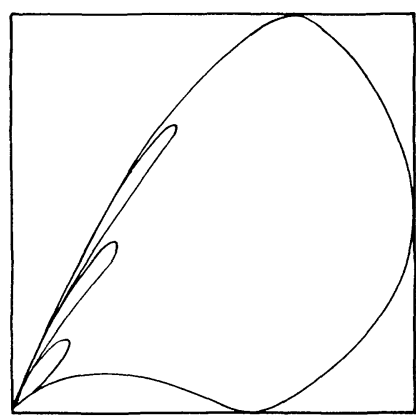

(c)

Fig. 3.3. a The invariant circle and the unstable manifold associated with the fixed point $(0,0)$ for the delayed logistic map when $a=2.09$. The computation was started near the fixed point $\frac{a-1}{a}(1,1)$ and the "spiral arms" show how the iterates approach the invariant circle. b Schematic drawing of the unstable manifold associated with $(0,0)$ for a near $a^{*}$. c Schematic drawing of the unstable manifold associated with $(0,0)$ for $a=a^{*}$

called a non-degenerate homoclinic point. If they intersect tangentially, then we call $x$ a point of homoclinic tangency.

For non-invertible maps it is possible that $x_{n}=z$ for all sufficiently large values of $n$. For example, for the map $F_{a}$ we have

$$
F_{a}(x, 0)=(0,0) \text { for all } x .
$$

The origin has one eigenvalue equal to 0 and the other equal to $a$, so that it is a saddle point for $a>1$. Its stable manifold is the $x$-axis while one branch of its unstable manifold points into the first quadrant. Pounder and Rogers have shown that, for $a=a^{*} \approx 2.2701$, the unstable manifold becomes tangent to the $x$-axis, and a point of homoclinic tangency develops [22] (see Fig. 3.3). It is exactly this critical 


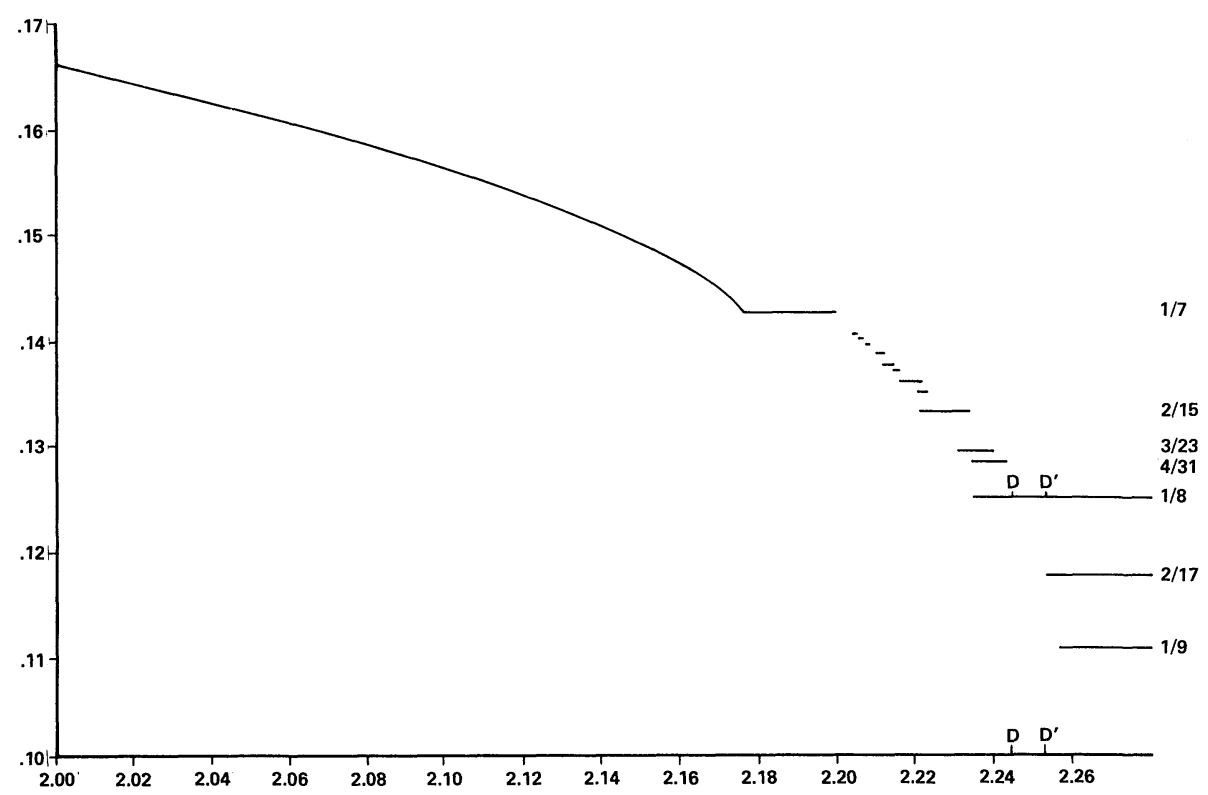

Fig. 3.4. Rotation number versus parameter $a$ for the delayed logistic map

value of $a$ which marks the end of the attractor. For $a<a^{*}$, one can construct an annular attractor block entirely within the first quadrant. For $a>a^{*}$, most points starting in the first quadrant eventually tend to infinity. Thus, once a point homoclinic to the origin appears, the invariant set we are studying ceases to be an attractor. However, it is the approach to this homoclinic tangency which seems to govern the transformation of the smooth invariant circle into a strange attractor.

An indication of the breakdown of the invariant circle as $a$ approaches $a^{*}$ can be seen in Fig. 3.4, which is a plot of the rotation number versus the parameter $a$. For $a<2.17$, the attractor is an invariant circle, and its rotation number is computed by approximating the limit (2.1) along a single orbit. We know from the general theory that this plot is the graph of a Cantor function, but we sketch it as a smooth curve, since that is how it looks at this scale. For $a>2.18$, we compute the rotation number by finding a periodic saddle, and we plot the parameter interval over which the periodic saddle is present.

When $a$ reaches approximately 2.177 , a $1 / 7$ resonance occurs and persists until $a$ is approximately 2.200 . During this resonance, the attractor remains topologically a circle, although it loses differentiability, as will be discussed in Sect. 8.

For $2.200<a<2.205$, the attractor still appears to be topologically a circle, but we are not confident of our resolution of this region. For $a>2.205$, there are many intervals of $a$ for which the rotation number is unique interspersed with intervals for which an infinite number of different rotation numbers exist. The last interval with a unique rotation number is approximately $(2.2414,2.2520)$, where the rotation number is $1 / 8$. The endpoints of this interval are marked $D$ and $D^{\prime}$ in Fig. 3.4. Beyond this interval, the rotation number of $1 / 8$ persists, while apparently 


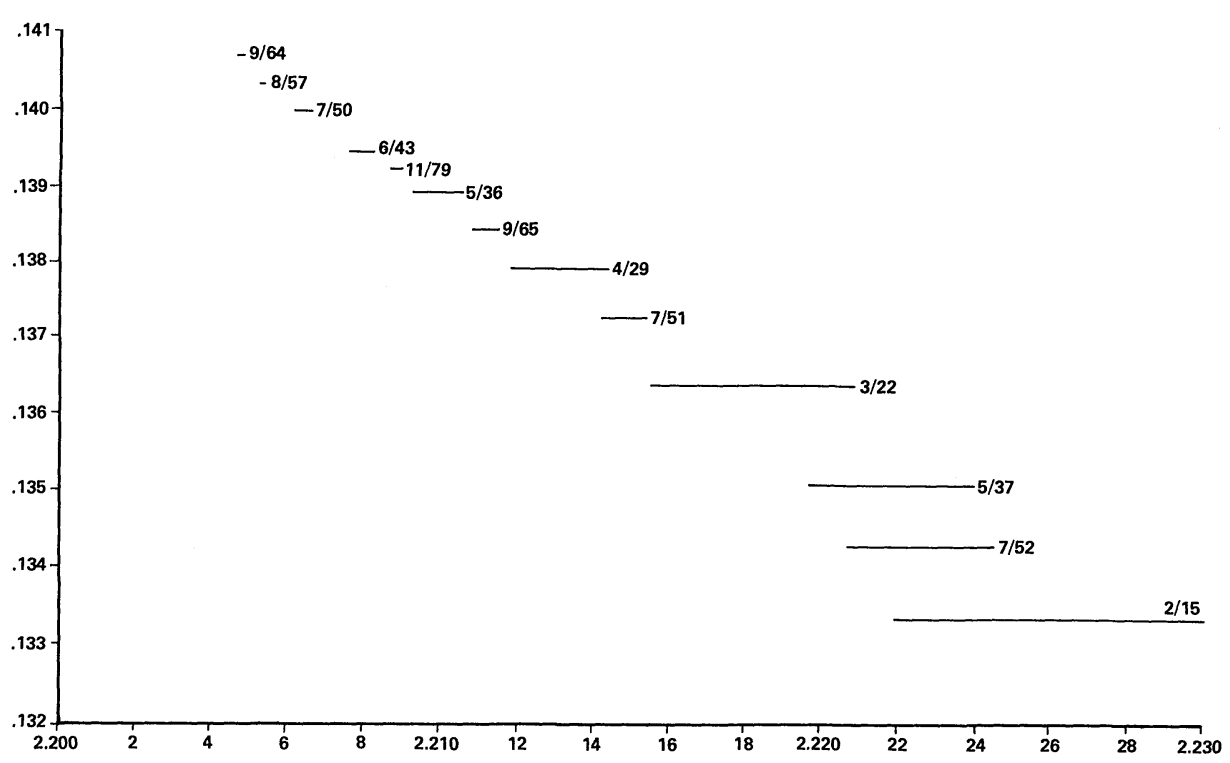

Fig. 3.5. Enlargement of a portion of Fig. 3.4

all the rotation numbers between $1 / 8$ and 0 appear in decreasing order as $a$ increases to $a^{*}$.

Some of the complexity can be seen in Fig. 3.5, which is a plot of some of the rotation numbers for $2.205<a<2.225$. The rotation number $2 / 15$ is unique in part of the interior of its parameter interval, but not unique near the endpoints of its interval. The same is true for rotation numbers $3 / 22,4 / 29$, and $5 / 36$. It appears that rotation number $6 / 43$ is unique near the left endpoint and in the interior of its parameter interval, but not near the right endpoint. It seems reasonable to conjecture that there are an infinite number of intervals of unique rotation number interspersed among an infinite number of intervals of nonunique rotation number.

The following fact about rational numbers is useful for thinking about rotation numbers. If $a / b$ and $c / d$ are rational numbers expressed in lowest terms, and if every rational number between $a / b$ and $c / d$ has a denominator larger than both $b$ and $d$, then $(a+c) /(b+d)$ is the unique rational number between $a / b$ and $c / d$ with the smallest denominator. For example, the lowest order resonance between $1 / 7$ and $1 / 8$ is $(1+1) /(7+8)=2 / 15$, between $1 / 7$ and $2 / 15$ is $3 / 22$, etc. This fact is closely related to Farey sequences [23]. The Farey sequence of order $N$ is the ordered sequence of all nonnegative reduced fractions between 0 and 1 whose denominators do not exceed $N$. The numbers $a / b$ and $c / d$ are two consecutive fractions in a Farey sequence. The number $(a+c) /(b+d)$ is called the "mediant" between $a / b$ and $c / d$.

To understand better the complexity of the rotation number structures indicated in Figs. 3.4 and 3.5, we introduce a second parameter into the delayed regulation model. Before introducing this new model, we first discuss Hopf bifurcation in two-parameter families. Our discussion follows closely Arnol'd [1]. 


\section{Hopf Bifurcation in Two-Parameter Families}

Throughout this section we use the complex numbers as a coordinate system on the real plane. A map on $R^{2}$ can be written as a function of one complex variable, and two real parameters can be written as a single complex parameter.

Consider the map from the complex plane to itself

$$
z \rightarrow f_{\mu}(z)=\mu z+O\left(|z|^{2}\right),
$$

where $z, \mu \in C$ and $\mu$ is the complex parameter. Note that $z=0$ is a fixed point of $f_{\mu}$, for all $\mu$. The eigenvalues at this fixed point are $\mu$ and $\bar{\mu}$. The above form for $f_{\mu}$ is general, since almost any two parameter family of maps having a fixed point with complex eigenvalues can be transformed to the form (4.1) as follows. First translate the fixed point to the origin. Then make a complex linear change of coordinates which diagonalizes the linear part of the map. Finally, introduce the eigenvalue at the origin as a new complex parameter. The only additional condition necessary is that the function which gives this eigenvalue in terms of the original parameters should be invertible.

The origin is stable for $|\mu|<1$ and unstable for $|\mu|>1$. We are interested in the bifurcation associated with the loss of stability occurring at $|\mu|=1$. To study this bifurcation we look at values of $\mu$ near $\mu_{0}$, a point on the unit circle. Certain low order resonances must be excluded to insure the bifurcation of an invariant circle. (For a discussion of the bifurcations near these resonances, see Arnol'd [1], Iooss [14], or Takens [29]). Thus we assume that

$$
\mu_{0}^{k} \neq 1, k=1,2,3,4 \text {. }
$$

We now write $f_{\mu}$ in its normal form,

$$
f_{\mu}(z)=\mu z+c(\mu) z|z|^{2}+O\left(|z|^{4}\right) .
$$

To insure that an attracting invariant circle bifurcates out of the fixed point for $|\mu|>1$, we assume that

$$
\operatorname{Re}\left(\bar{\mu}_{0} c\left(\mu_{0}\right)\right)<0 \text {. }
$$

Under these assumptions, the Hopf bifurcation theorem for maps states that, for $\mu$ near $\mu_{0}$, with $|\mu|>1$, the map $f_{\mu}$ has an attracting invariant circle surrounding $z=0$ $[1,29]$.

Recall that resonance occurs on the invariant circle if there is a pair of periodic orbits, one consisting of saddles and the other of sinks. We say that a $p / q$ resonance occurs if the rotation number of these periodic orbits is $p / q$.

Now consider $\mu_{0}=e^{i 2 \pi p / q}, q \geqq 5$. The values of $\mu$ for which the $p / q$ resonance occurs lie in a horn emanating from $\mu_{0}$. Specifically, let

$$
\mu=(1+\sigma+i \tau) \mu_{0} .
$$

Resonance occurs in a region

$$
|\tau-\alpha \sigma| \leqq \beta \sigma^{(q-2) / 2}+O\left(\sigma^{q / 2}\right),
$$

where the real coefficients $\alpha$ and $\beta$ depend only on the terms up to order $q$ in the expansion of $f_{\mu}$ [1] (see Fig. 4.1). 


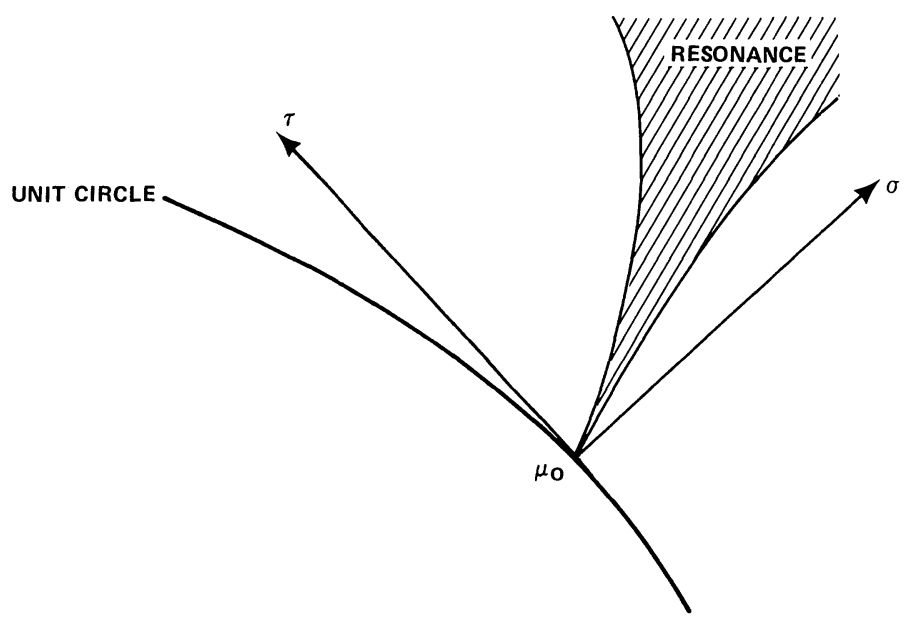

Fig. 4.1. Schematic drawing of a resonance horn emerging from the unit circle in the complex $\mu$-plane

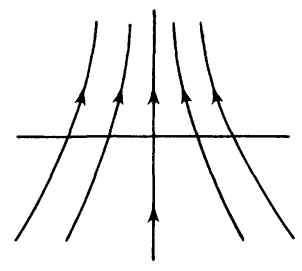

(a) $\epsilon<0$

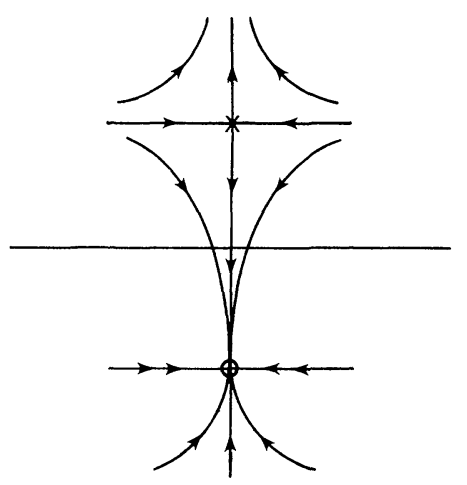

(c) $\epsilon>0$

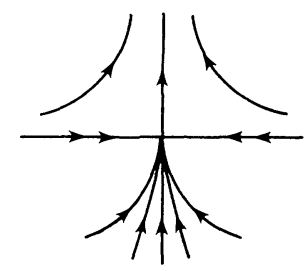

(b) $\epsilon=\mathbf{0}$

Fig. 4.2a-c. Creation of saddle-sink pairs for the model map (4.2). a No fixed point for $\varepsilon<0$. b One fixed point, a saddle-node, for $\varepsilon=0$. c Saddle-sink pair for $\varepsilon>0$

The boundaries of the horn correspond to "saddle-nodes." The creation of a saddle-sink pair through a saddle-node is best seen in the following simple model near $(x, y)=(0,0)$ :

$$
(x, y) \rightarrow\left(r x, y-\varepsilon+y^{2}\right),
$$

where $r$ is a fixed constant with $0<r<1$, and where $\varepsilon$ is a parameter. For $\varepsilon<0$ there is no fixed point, for $\varepsilon=0$ there is one fixed point, a saddle-node, and for $\varepsilon>0$ there 


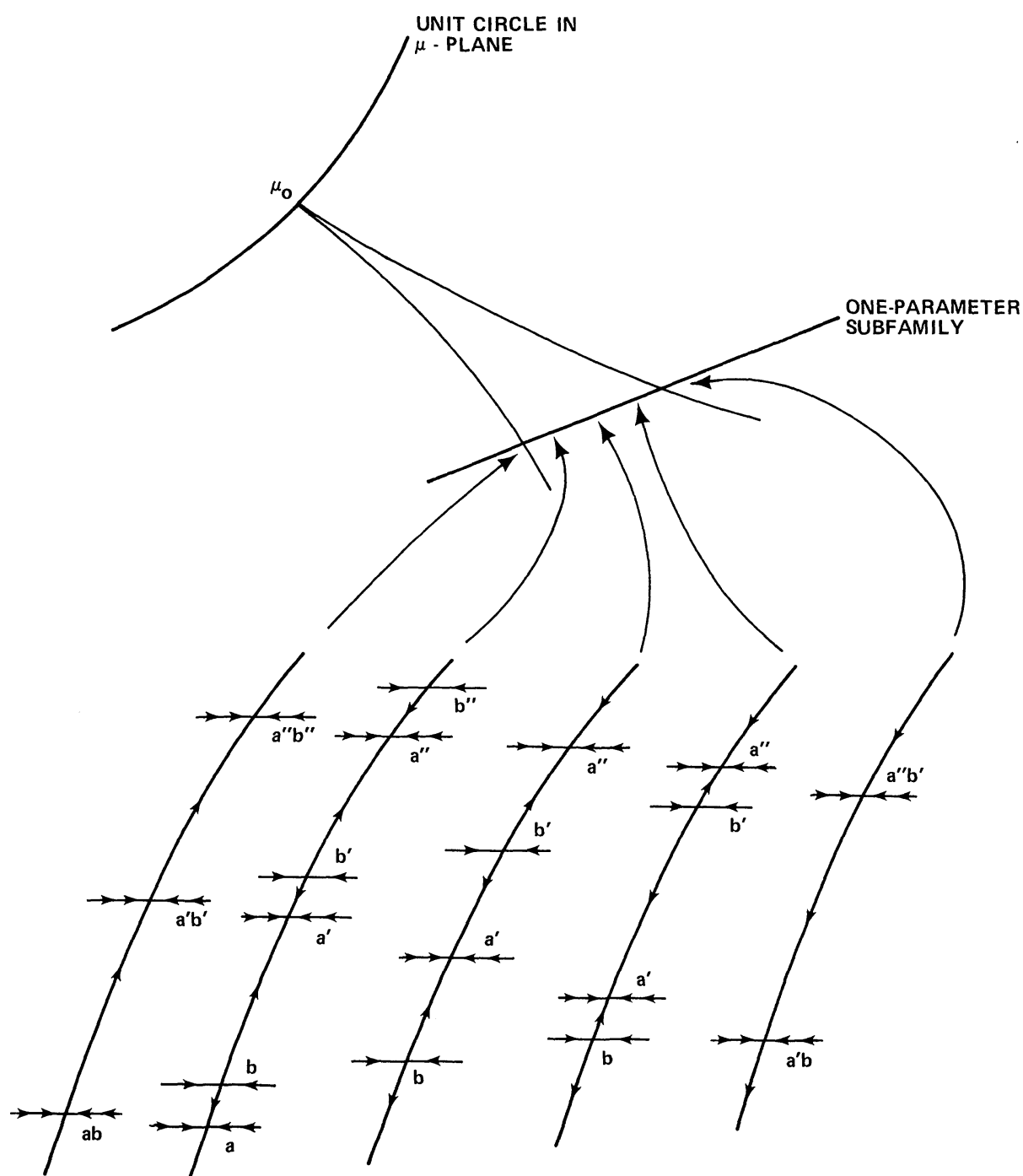

Fig. 4.3. A typical transition from saddle-node to saddle-sink pair and back to saddle-node along a one-parameter section through a resonance horn

are two fixed points, a saddle-sink pair (see Fig. 4.2). The saddle and sink are at the points $x=0, y= \pm \sqrt{\varepsilon}$, growing out of the saddle-node as $\varepsilon$ passes through 0 .

When $\varepsilon$ is positive and small enough, there is a distinguished manifold associated with the sink. This manifold is the set $\{y=-\sqrt{\varepsilon}\}$ and is indicated with double arrows in Fig. 4.2c. It is the set of points which approach the sink at the exponential rate $r^{n}$. All other points approaching the sink approach at the rate $(1-2 \sqrt{\varepsilon})^{n}$, the slower exponential rate given by the larger eigenvalue. Even with the addition of the higher order terms, the fast rate distinguishes a smooth curve, called the strong stable manifold of the sink. 
There are also distinguished manifolds for the saddle-node. The strong stable manifold is the set of points approaching the fixed point at an exponential rate. This manifold is the $x$-axis for the above model and is indicated with double arrows in Fig. 4.2b. The other distinguished manifold is the unstable manifold, the set of points approaching the saddle-node backward in time. This manifold is the positive $y$-axis for the above model.

We now return to our discussion of the resonance horn. Consider a oneparameter subfamily passing across the horn, as shown in Fig. 4.3. When the parameter is outside the horn, no periodic point of rotation number $p / q$ is present. When the parameter encounters the boundary of the horn, $q$ saddle-nodes appear on the invariant circle. These points are the iterates of a periodic point of period $q$ and are labeled $a b, a^{\prime} b^{\prime}$, and $a^{\prime \prime} b^{\prime \prime}$ in Fig. 4.3. As the parameter passes into the interior of the horn, the saddle-nodes bifurcate, forming saddle-sink pairs. In Fig. 4.3 the sinks are labeled $a, a^{\prime}$, and $a^{\prime \prime}$, while the corresponding saddles are labeled $b, b^{\prime}$, and $b^{\prime \prime}$. There are now two periodic orbits on the invariant circle, one consisting of the saddles and the other of the sinks. The $q^{\text {th }}$ iterate of the map has $2 q$ fixed points, alternating between saddles and sinks around the invariant circle, as illustrated in Fig. 4.3. As the parameter continues to move across the horn, the saddles and sinks move apart. As the parameter approaches the other boundary, these points form different saddle-sink pairs. In Fig. 4.3 the saddle labeled $b$ pairs with the sink labeled $a^{\prime}$, while the saddle labeled $b^{\prime}$ pairs with the sink labeled $a^{\prime \prime}$. When the parameter encounters the other boundary of the horn, these new saddlesink pairs combine to form the saddle-nodes labeled $a^{\prime} b$ and $a^{\prime \prime} b^{\prime}$.

\section{A Modified Model of Delayed Regulation}

In order to apply the Arnol'd theory to the delayed regulation map, we embed it in a two parameter family of maps. Such an embedding can be accomplished in an infinite variety of ways, some of which may yield models of interesting population interactions. However, we have ignored these considerations and instead have chosen our two parameter family on the basis of simplicity and computational convenience. Specifically, we consider the family of maps,

$$
F_{(a, b)}(x, y)=(y+b x, a y(1-x)) .
$$

Note that this map reduces to the model of Sect. 3 when $b=0$, i.e., that $F_{(a, 0)} \equiv F_{a}$.

The map has a fixed point at

$$
x=\frac{a-1}{a}, y=(1-b) \frac{a-1}{a} .
$$

The Jacobian matrix at this fixed point is

$$
\left[\begin{array}{cc}
b & 1 \\
-(1-b)(a-1) & 1
\end{array}\right]
$$

If the parameters satisfy $5 / 4<a<3,0 \leqq b<1$, then the eigenvalues of this matrix are complex. Thus in this parameter range the map (5.1) can be written in the complex form (4.1) and studied by the machinery of Sect. 4 . The eigenvalues are 


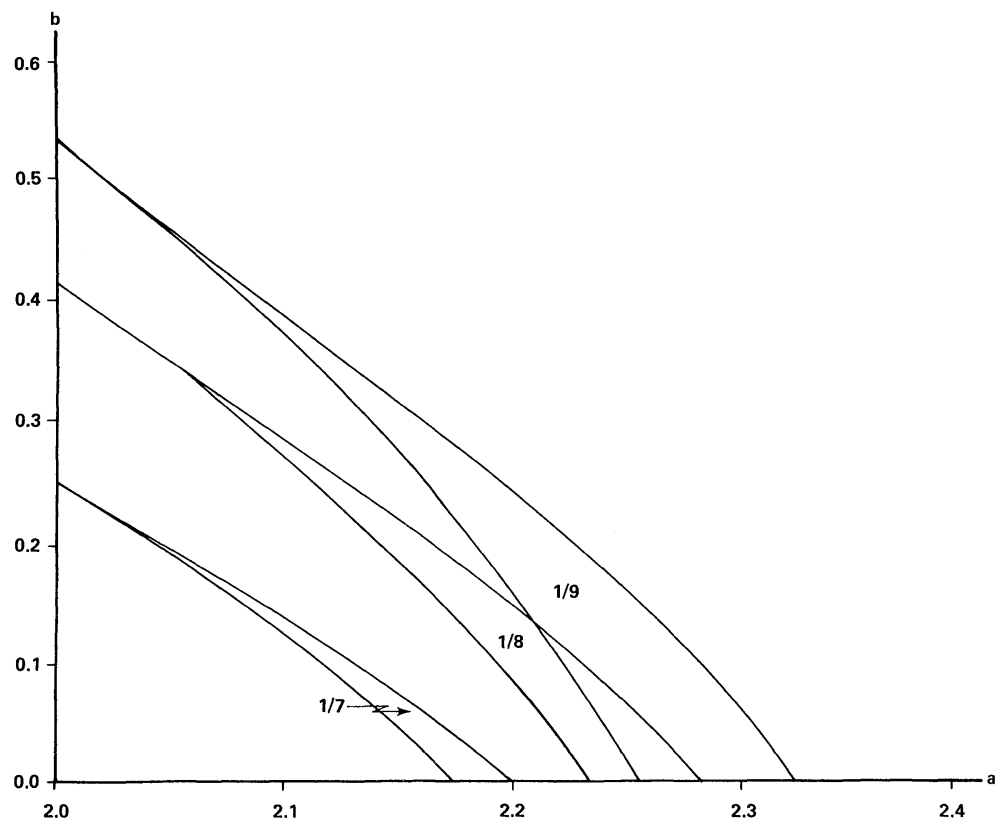

Fig. 5.1. Resonance horns for the two-parameter version of the delayed logistic map, Eq. (5.1). Horns corresponding to rotation numbers $1 / 7,1 / 8$, and $1 / 9$ are shown to scale

inside the unit circle for $a<2$ and outside the unit circle for $a>2$, independent of $b$. When $a=2$, the eigenvalues are $e^{ \pm i \alpha}$, where

$$
\cos \alpha=\frac{1+b}{2} \text {. }
$$

Therefore the $p / q$ resonance horn bifurcates from the line $a=2$ at the value of $b$ which satisfies

$$
\cos (2 \pi p / q)=\frac{1+b}{2}
$$

We have computed various resonance horns, and the results of the computations for three of them are shown in Fig. 5.1. These horns extend well into the region where the attractor is no longer a circle, and hence where the machinery of Sect. 4 no longer applies. The definition that we used to compute the horns is this: a point $(a, b)$ is in the $p / q$ resonance horn if $F_{(a, b)}$ has a periodic saddle of rotation number $p / q$. A boundary point of the horn corresponds to a periodic saddle-node of the same rotation number. These boundary points were computed using Newton's method to solve a system of equations containing the condition that 1 is an eigenvalue of the periodic point.

The rotation number graphs in Figs. 3.4 and 3.5 show the intersection of these horns with the $a$-axis, i.e., with the one parameter subfamily given by $b=0$. Overlapping rotation numbers correspond to overlapping resonance horns. The 
$1 / 7$ resonance horn does not intersect any other horn along the $a$-axis. However, the $1 / 8$ and $1 / 9$ resonance horns do intersect, corresponding to the overlap of these rotation numbers in Fig. 3.4.

We now wish to study in detail the behavior of the attractor for parameter values inside the resonance horns. We shall return at the end of Sect. 7 to a further discussion of our computer simulations of the map $F_{(a, b)}$. First we prove some general results about rotation numbers for annular attractor blocks.

\section{Unique Rotation Numbers}

When the parameter values are inside the $p / q$ resonance horn and near its tip, the attractor is a smooth circle in resonance, as discussed in Sect. 4. For these parameter values, every point in $A$ has rotation number $p / q$. As the parameter point moves inside the resonance horn away from the tip, it is possible for the attractor to distort until it ceases to be a circle. This process will be discussed in Sect. 8, but for now we wish to describe sufficient conditions for the rotation number to be unique.

We begin by returning to the concept of rotation number. Throughout this section we assume that $B \subset R^{2}$ is an annulus, that $F: B \rightarrow F(B) \subset B$ is a diffeomorphism, and that $B$ is an attractor block with associated attractor $A$. Without loss of generality we can assume that

$$
B=\{z \in C: 1 \leqq|z| \leqq 2\} ;
$$

otherwise, we can map $B$ onto this set with a diffeomorphism. Let

$$
\hat{B}=\left\{(r, \theta) \in R^{2}: 1 \leqq r \leqq 2\right\},
$$

and let

$$
\tau: \hat{B} \rightarrow B:(r, \theta) \rightarrow r e^{i \theta}
$$

be the standard covering projection using polar coordinates. Now let $\hat{F}$ be a lift of $F$ to $\hat{B}$, i.e. let $\hat{F}: \hat{B} \rightarrow \hat{B}$ be a continuous map such that

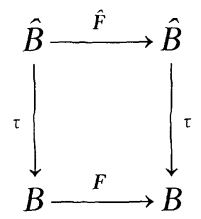

commutes. Note that $\hat{F}$ is unique up to translation by a multiple of $2 \pi$ in the angular variable $\theta$.

Now let arg denote the projection of $\hat{B}$ onto the angular variable, i.e.

$$
\arg (r, \theta)=\theta \text {. }
$$

We always assume that $F$ has degree 1, i.e. that

$$
\arg \hat{F}(r, \theta+2 \pi)=2 \pi+\arg \hat{F}(r, \theta) \quad \text { for all }(r, \theta) \in \hat{B} .
$$




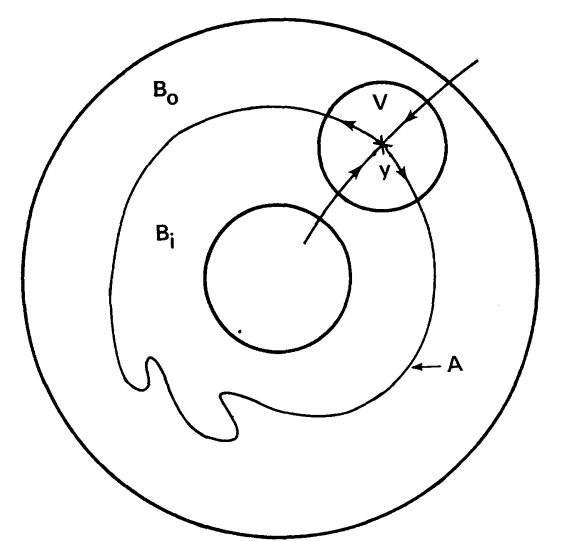

Fig. 6.1. Schematic drawing of the attractor block $B$ divided into two components $B_{o}$ and $B_{i}$ by the attractor $A$. Also shown is the local structure of the attractor and the stable manifold in a neighborhood $V$ of a periodic saddle point $y$ (cf. Lemma 1)

Definition. Let $z \in B$, and choose $(r, \theta) \in \hat{B}$ such that $\tau(r, \theta)=z$. If the following limit exists, it is called the rotation number of $z$ :

$$
\varrho(z)=\lim _{n \rightarrow \infty} \frac{\arg \hat{F}^{n}(r, \theta)-\theta}{2 \pi n} .
$$

Note that, for any given lift $\hat{F}$ and any given $z \in B, \arg \hat{F}^{n}(r, \theta)-\theta$ is independent of the choice of $\theta$. Note also that, if a different lift $\hat{F}$ is chosen, $\varrho(z)$ can change only by an integer. Thus $\varrho(z)$ is well-defined modulo 1 . Henceforth we fix a lift $\hat{F}$ and compute all rotation numbers with respect to that lift.

We are now ready to state the main result of this section. First note that $A$ is a compact connected set which separates the annulus $B$ into two components: $B_{i}$, containing the inside boundary of $B$, and $B_{o}$, containing the outside boundary (see Fig. 6.1).

Theorem I. Suppose that $F$ satisfies the following three hypotheses:

(a) There exists a periodic saddle point $y \in A$ with positive eigenvalues.

(b) $W^{s}\left(y, F^{q}\right) \cap A=\{y\}$, where $q$ is the period of $y$.

(c) One branch of $W^{s}\left(y, F^{q}\right)$ intersects only $B_{i}$, while the other branch intersects only $B_{o}$.

Then $\varrho(z)=\varrho(y)$ for all $z \in B$.

Note that the hypotheses of Theorem I are satisfied near the tip of the $p / q$ resonance horn where $A$ is a smooth circle in resonance.

Before we prove this theorem, it is necessary to describe the local structure of $A$ near $y$. To this end, we define the local stable and unstable manifolds for a fixed point. Let $f: R^{2} \rightarrow R^{2}$ be a diffeomorphism and let $z \in R^{2}$ be a fixed point with saddle structure. Let $U$ be an open neighborhood of $z$. Then the local unstable manifold of $z$ is

$$
W_{\mathrm{loc}}^{u}(z, f, U) \equiv\left\{x \in U: f^{n}(x) \in U \quad \text { for all } \quad n \leqq 0 \quad \text { and } \quad f^{n}(x) \rightarrow z \quad \text { as } \quad n \rightarrow-\infty\right\},
$$


with a similar definition for the local stable manifold $W_{\text {loc }}^{s}(z, f, U)$. The stable manifold theorem states that there exists a $U$ such that $W_{\text {loc }}^{u}(z, f, U)$ and $W_{\text {loc }}^{s}(z, f, U)$ are smooth embedded arcs [12]. Henceforth we fix such a $U$. Note that the global unstable manifold as previously defined satisfies

$$
W^{u}(z, f)=\bigcup_{n \geqq 0} f^{n}\left(W_{\mathrm{loc}}^{u}(z, f, U)\right) .
$$

Lemma 1. Let $F$ satisfy hypotheses (a) and (b) of Theorem I. Then there exists an open set $V$, with $y \in V \subset U$, and such that

$$
A \cap V=W_{\mathrm{loc}}^{u}\left(y, F^{q}, U\right) \cap V .
$$

Proof. Let the eigenvalues of $D F^{q}(y)$ be $\mu$ and $v$ satisfying $0<v<1<\mu$. By Hartman's linearization theorem [12], we may choose a $C^{0}$ coordinate system in a neighborhood of $y$ such that $f \equiv F^{q}$ can be written

$$
f(\xi, \eta)=(\mu \xi, v \eta)
$$

We may assume without loss of generality that this coordinate system is valid for

$$
U=\{(\xi, \eta):|\xi|<1,|\eta|<1\} .
$$

In these coordinates, $y=(0,0)$,

$$
W_{\mathrm{loc}}^{u}(y, f, U)=\{(\xi, \eta) \in U: \eta=0\}
$$

and

$$
W_{\text {loc }}^{\text {s }}(y, f, U)=\{(\xi, \eta) \in U: \xi=0\} .
$$

Assume that the conclusion of the lemma is false, i.e., assume that a sequence $\zeta_{k}=\left(\xi_{k}, \eta_{k}\right) \in A$ exists such that $\zeta_{k} \notin W_{\text {loc }}^{u}(y, f, U)$ for all $k$ and such that $\zeta_{k} \rightarrow(0,0)$ as $k \rightarrow \infty$. By choosing a subsequence, if necessary, we may assume that the entire sequence lies in a single quadrant, which we may assume without loss of generality is the first quadrant. Since $\eta_{k}>0$, there is a unique integer $n_{k}$ satisfying

$$
v \leqq \eta_{k} v^{-n_{k}}<1 \text { for each } k \text {. }
$$

Choose a subsequence $k(i)$ so that

$$
\eta_{k(i)} v^{-n_{k(i)}} \rightarrow \eta^{*} \in[v, 1] \quad \text { as } \quad i \rightarrow \infty
$$

Relabel the sequences so that $n_{k(i)}$ becomes $n_{i}$ and $\zeta_{k(i)}=\left(\xi_{k(i)}, \eta_{k(i)}\right)$ becomes $\zeta_{i}=\left(\xi_{i}, \eta_{i}\right)$. Since $A$ is invariant, $f^{-n_{i}}\left(\zeta_{i}\right) \in A$ for all $i$. But

$$
f^{-n_{i}}\left(\zeta_{i}\right)=\left(\mu^{-n_{i}} \xi_{i}, v^{-n_{i}} \eta_{i}\right) \rightarrow\left(0, \eta^{*}\right) \quad \text { as } \quad i \rightarrow \infty .
$$

Since $A$ is closed, $\left(0, \eta^{*}\right) \in A$. But $\left(0, \eta^{*}\right) \in W_{\text {loc }}^{s}(y, f, U)$, which contradicts hypothesis (b) and establishes the lemma.

Now that we have determined the local structure of $A$ near $y$, we can show that the rotation number is unique on $A$. First we establish a formula for the rotation number. 
Lemma 2. Fix an integer $q>0$, fix $z \in B$, and let $\hat{z} \in \hat{B}$ satisfy $\tau(\hat{z})=z$. Then

$$
\varrho(z)=\lim _{n \rightarrow \infty} \frac{\arg \hat{F}^{n q}(\hat{z})}{2 \pi n q} .
$$

By the above formula we mean the following. The limit exists if and only if $\varrho(z)$ exists. If $\varrho(z)$ exists, then it equals the limit.

Proof. Set

$$
\hat{z}_{k}=\hat{F}^{k}(\hat{z})
$$

For $n q \leqq k<n q+q$, write

$$
\arg \hat{z}_{k}-\arg \hat{z}=\arg \hat{z}_{n q}-\arg \hat{z}+R_{k},
$$

where

$$
R_{k}=\sum_{l=0}^{k-1-n q}\left(\arg \hat{z}_{k-l}-\arg \hat{z}_{k-1-l}\right)
$$

Since $F$ has degree 1 , the function

$$
\hat{B} \rightarrow R^{1}:(r, \theta) \rightarrow \arg \hat{F}(r, \theta)-\theta
$$

is periodic in $\theta$ and is therefore bounded on $\hat{B}$. In particular,

$$
|\arg \hat{F}(r, \theta)-\arg (r, \theta)| \leqq K \text { for all }(r, \theta) \in \hat{B},
$$

so that $\left|R_{k}\right|<q K$. Consequently we obtain the estimate

$$
\frac{\arg \hat{z}_{n q}-\arg \hat{z}}{2 \pi n q}\left(1-\frac{q}{k}\right)-\frac{q K}{2 \pi k} \leqq \frac{\arg \hat{z}_{k}-\arg \hat{z}}{2 \pi k} \leqq \frac{\arg \hat{z}_{n q}-\arg \hat{z}}{2 \pi n q}+\frac{q K}{2 \pi k},
$$

from which it follows that

$$
\varrho(z)=\lim _{n \rightarrow \infty} \frac{\arg \hat{z}_{n q}-\arg \hat{z}}{2 \pi n q}
$$

if either side of the equality exists.

Lemma 3. Let $F$ satisfy the hypotheses of Theorem I. Then $\varrho(z)=\varrho(y)$ for all $z \in A$. Proof. Let $\hat{Y}$ be the lift of $y$ to $\hat{B}$, i.e., let

$$
\hat{Y}=\tau^{-1}(y)=\left\{\ldots, \hat{y}_{-1}, \hat{y}_{0}, \hat{y}_{1}, \ldots\right\},
$$

where we have numbered the points in $\hat{Y}$ so that

$$
\arg \hat{y}_{k+1}=\arg \hat{y}_{k}+2 \pi \quad \text { for all integers } k .
$$

We see that there is an integer $p$ satisfying

$$
\hat{F}^{q}\left(\hat{y}_{k}\right)=\hat{y}_{k+p} \text { for all } k,
$$

which gives us the rotation number $\varrho(y)=p / q$. Let $\hat{A}$ be the lift of $A$ to $\hat{B}$, i.e. let

$$
\hat{A}=\tau^{-1}(A) \subset \hat{B} \text {. }
$$




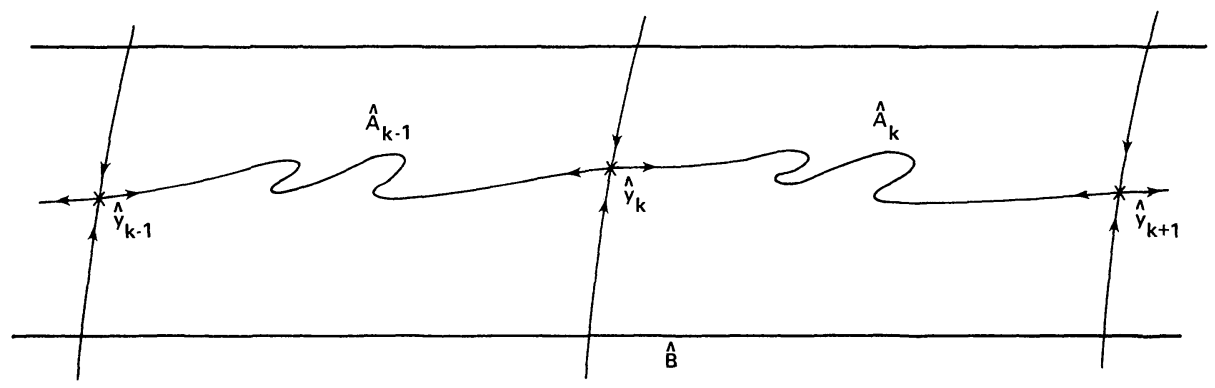

Fig. 6.2. The components $\hat{A}_{k}$ of the lift of $A-\{y\}$

By Lemma 1, the attractor $A$ in a neighborhood of the point $y$ consists of a smooth arc, the local unstable manifold of $y$. By hypothesis (c), one branch of the stable manifold of $y$ lies in $B_{i}$ while the other branch lies in $B_{o}$, which implies that $A$ separates $B_{i}$ from $B_{o}$ locally near $y$. Therefore we can construct a smooth arc $C$ which connects the inner boundary of $B$ to the outer boundary and which intersects $A$ only at $y$. The lift $\hat{C}$ of $C$ to $\hat{B}$ separates $\hat{B}$ into bounded components, each projecting onto $B-C$. Since $C$ intersects $A$ only at $y, \hat{A}-\hat{C}=\hat{A}-\hat{Y}$ separates into bounded components, each projecting onto $A-\{y\}$. Label these components $\hat{A}_{k}$ so that

$$
\operatorname{cl}\left(\hat{A}_{k}\right)=\hat{A}_{k} \cup\left\{\hat{y}_{k}, \hat{y}_{k+1}\right\},
$$

where "cl" denotes topological closure (see Fig. 6.2). For a set $\hat{S} \subset \hat{B}$ and a real number $\alpha$, we use the notation

$$
\hat{S}+\alpha \equiv\{(r, \theta+\alpha) \in \hat{B}:(r, \theta) \in \hat{S}\} .
$$

Note that

$$
\hat{A}_{k}=\hat{A}_{0}+2 \pi k
$$

and that

$$
\hat{F}^{n q}\left(\hat{A}_{k}\right)=\hat{A}_{k+n p} .
$$

Since $\hat{A}_{0}$ is bounded, there exist numbers $b_{1}$ and $b_{2}$ such that

$$
b_{1} \leqq \arg (\hat{w}) \leqq b_{2} \text { for all } \hat{w} \in \hat{A}_{0} .
$$

Now let $\hat{z} \in \hat{A}_{0}, \tau(\hat{z})=z$. Then

$$
\hat{F}^{n q}(\hat{z}) \in \hat{A}_{n p}=\hat{A}_{0}+2 \pi n p,
$$

so

$$
\begin{aligned}
& 2 \pi n p+b_{1} \leqq \arg \hat{F}^{n q}(\hat{z}) \leqq 2 \pi n p+b_{2} \\
& \frac{p}{q}+\frac{b_{1}}{2 \pi n q} \leqq \frac{\arg \hat{F}^{n q}(\hat{z})}{2 \pi n q} \leqq \frac{p}{q}+\frac{b_{2}}{2 \pi n q}
\end{aligned}
$$

Hence, by Lemma 2, $\varrho(z)=p / q$, which completes the proof. 
It remains to show that the rotation number is $p / q$ for every orbit in $B$. We shall use the concepts of pseudo-orbits and shadows [3], ideas which will be useful again in Sect. 7. Recall that an orbit is a bi-infinite sequence of points $x_{i} \in R^{2}$ such that

$$
F\left(x_{i}\right)=x_{i+1} \text { for all } i .
$$

In contrast, we define a pseudo-orbit as follows.

Definition. Let $\varepsilon \geqq 0$. An $\varepsilon$-pseudo-orbit is a bi-infinite sequence of points $z_{i} \in R^{2}$ such that

$$
d\left(F\left(z_{i}\right), z_{i+1}\right) \leqq \varepsilon \quad \text { for all } i .
$$

Of course, a pseudo-orbit may be defined only for $i \geqq 0$ or $i \leqq 0$, in which case it should be called a "semi-pseudo-orbit." However, we shall refer to them all as simply "pseudo-orbits."

The concept of a pseudo-orbit is useful in computational work, since a computer in fact follows an $\varepsilon$-pseudo-orbit, where $\varepsilon$ is determined by the arithmetic of the particular machine.

As we shall see in Sect. 7, it is often possible to find a true orbit very close to a pseudo-orbit. We therefore define a "shadow" of a pseudo-orbit as follows.

Definition. Let $\delta \geqq 0$. The orbit $\left\{x_{i}\right\}$ is said to the a $\delta$-shadow of the pseudo-orbit $\left\{z_{i}\right\}$ if

$$
d\left(x_{i}, z_{i}\right) \leqq \delta \quad \text { for all } i
$$

For convenience, we always use the Euclidean metric on $\hat{B}$ and the metric on $B$ induced by the covering map $\tau: \hat{B} \rightarrow B$. Then, if $\varepsilon<\pi$, every $\varepsilon$-pseudo-orbit $\left\{z_{i}\right\}$ in $B$ lifts to a unique $\varepsilon$-pseudo-orbit $\left\{\hat{z}_{i}\right\}$ in $\hat{B}$, once $\hat{z}_{0}$ is chosen. In addition, we can define the rotation number of the pseudo-orbit $\left\{z_{i}\right\}$ as the following limit, if it exists :

$$
\varrho\left(\left\{z_{i}\right\}\right)=\lim _{n \rightarrow \infty} \frac{\arg \hat{z}_{n}-\arg \hat{z}_{0}}{2 \pi n} .
$$

Henceforth, we shall always assume that $\varepsilon<\pi$ for every $\varepsilon$-pseudo-orbit. Furthermore, we shall always assume that $\delta<\pi$ for every $\delta$-shadow. This restriction assures that, if $\left\{x_{i}\right\}$ is a $\delta$-shadow of an $\varepsilon$-pseudo-orbit $\left\{z_{i}\right\}$, there exist lifts $\left\{\hat{x}_{i}\right\}$ and $\left\{\hat{z}_{i}\right\}$ such that $\left\{\hat{x}_{i}\right\}$ is a $\delta$-shadow of $\left\{\hat{z}_{i}\right\}$ in $\hat{B}$. One easily sees that the orbit $\left\{x_{i}\right\}$ and the pseudo-orbit $\left\{z_{i}\right\}$ share rotation numbers. That is, if either rotation number exists, so does the other, and they are equal.

The proof of Theorem I now proceeds as follows. First we establish that every pseudo-orbit in $A$ has rotation number $p / q$. Then we prove that every orbit in $B$ eventually shadows some pseudo-orbit in $A$ and hence also has rotation number $p / q$. We begin by proving the following analog of Lemma 2.

Lemma 4. Fix integers $q>0$ and $m$, and assume that $\varepsilon<\pi$. Let $\left\{z_{i}\right\}$ be an $\varepsilon$-pseudoorbit in $B$ and let $\left\{\hat{z}_{i}\right\}$ be a lift to $\hat{B}$. Then

$$
\varrho\left(\left\{z_{i}\right\}\right)=\lim _{n \rightarrow \infty} \frac{\arg \hat{z}_{n q+m}}{2 \pi(n q+m)} .
$$


Proof. For $n q+m \leqq k<n q+m+q$, write

$$
\arg \hat{z}_{k}-\arg \hat{z}=\arg \hat{z}_{n q+m}-\arg \hat{z}+R_{k},
$$

where

$$
R_{k}=\sum_{l=0}^{k-1-n q-m}\left(\arg \hat{z}_{k-l}-\arg \hat{z}_{k-1-l}\right) .
$$

In view of (6.1) and the definition of $\varepsilon$-pseudo-orbit we have $\left|R_{k}\right|<(K+\varepsilon) q$. Thus we have the estimate

$$
\frac{\arg \hat{z}_{n q+m}-\arg \hat{z}}{2 \pi(n q+m)}\left(1-\frac{q}{k}\right)-\frac{q(K+\varepsilon)}{2 \pi k} \leqq \frac{\arg \hat{z}_{k}-\arg \hat{z}}{2 \pi k} \leqq \frac{\arg \hat{z}_{n q+m}-\arg \hat{z}}{2 \pi(n q+m)}+\frac{q(K+\varepsilon)}{2 \pi k},
$$

from which the assertion follows easily.

By analogy with the proof of Lemma 3, we also have:

Lemma 5. Let $F$ satisfy the hypotheses of Theorem I, let $\hat{A}$ and $\left\{\hat{A}_{k}\right\}$ be as in the proof of Lemma 3 , and let $q, m, \varepsilon,\left\{z_{i}\right\}$, and $\left\{\hat{z}_{i}\right\}$ satisfy the hypotheses of Lemma 4 . Suppose that

$$
\hat{z}_{n q+m} \in \hat{A}_{n p} \text { for all } n \geqq 0 \text {. }
$$

Then

$$
\varrho\left(\left\{z_{i}\right\}\right)=p / q
$$

Proof. Let $b_{1}$ and $b_{2}$ be defined by (6.2). Then

$$
\begin{gathered}
2 \pi n p+b_{1} \leqq \arg \hat{z}_{n q+m} \leqq 2 \pi n p+b_{2}, \\
\frac{2 \pi n p+b_{1}}{2 \pi(n q+m)} \leqq \frac{\arg \hat{z}_{n q+m}}{2 \pi(n q+m)} \leqq \frac{2 \pi n p+b_{2}}{2 \pi(n q+m)} .
\end{gathered}
$$

Hence, by Lemma $4, \varrho\left(\left\{z_{i}\right\}\right)=p / q$, which completes the proof.

We shall need the following estimate on pseudo-orbits.

Lemma 6. For each integer $n>0$ and each $\varepsilon>0$, there exists $\gamma_{n}(\varepsilon)>0$ such that, for every $\varepsilon$-pseudo-orbit $\left\{z_{i}\right\}$ in $B$,

$$
d\left(F^{n}\left(z_{k}\right), z_{k+n}\right) \leqq \gamma_{n}(\varepsilon) \text { for all } k .
$$

Furthermore, $\gamma_{n}(\varepsilon) \rightarrow 0$ as $\varepsilon \rightarrow 0$.

Proof. Since $F$ is uniformly continuous on $B$, there exists a $\mu(\varepsilon)$ such that $\mu(\varepsilon) \rightarrow 0$ as $\varepsilon \rightarrow 0$ and

$$
d\left(\xi_{1}, \xi_{2}\right)<\varepsilon \text { implies } d\left(F\left(\xi_{1}\right), F\left(\xi_{2}\right)\right)<\mu(\varepsilon) .
$$

Now define $\gamma_{n}(\varepsilon)$ by

$$
\begin{aligned}
\gamma_{1}(\varepsilon) & =\varepsilon \\
\gamma_{n+1}(\varepsilon) & =\mu\left(\gamma_{n}(\varepsilon)\right)+\varepsilon, \quad \text { for } n \geqq 1 .
\end{aligned}
$$




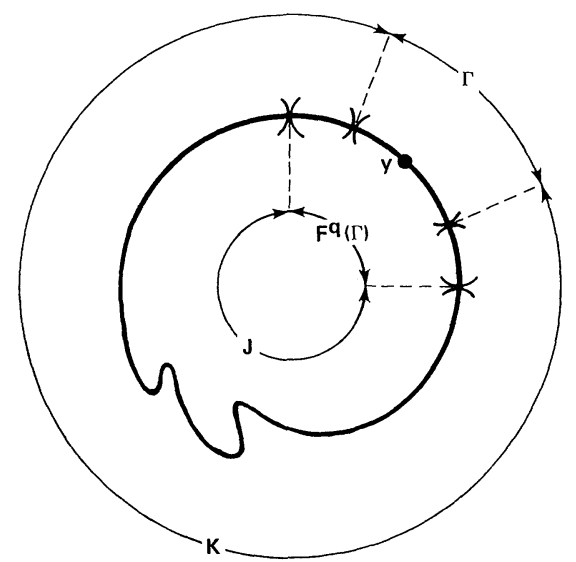

Fig. 6.3. The arc $\Gamma$ and the sets $K=A-\operatorname{cl}(\Gamma), F^{q}(\Gamma)$, and $J=F^{q}(K)$

The proof is completed using induction on $n$ and the following inequality

$$
\begin{aligned}
d\left(F^{n+1}\left(z_{k}\right), z_{k+n+1}\right) & \leqq d\left(F^{n+1}\left(z_{k}\right), F\left(z_{k+n}\right)\right)+d\left(F\left(z_{k+n}\right), z_{k+n+1}\right) \\
& \leqq \mu\left(\gamma_{n}(\varepsilon)\right)+\varepsilon=\gamma_{n+1}(\varepsilon) .
\end{aligned}
$$

We are now ready to prove that pseudo-orbits in $A$ all have rotation number $p / q$.

Lemma 7. Let $F$ satisfy the hypotheses of Theorem I. Then, for sufficiently small $\varepsilon>0$, every $\varepsilon$-pseudo-orbit in $A$ has rotation number $p / q$.

Proof. Let $\Gamma$ be a open arc such that

$$
y \in \Gamma \subset W_{\text {loc }}^{u}\left(y, F^{q}, U\right)
$$

and

$$
d(y, x)<\delta<\pi \text { for all } x \in \Gamma .
$$

By the definition of the local unstable manifold,

$$
\operatorname{cl}\left(F^{-q}(\Gamma)\right) \subset \Gamma, \text { so } \operatorname{cl}(\Gamma) \subset F^{q}(\Gamma) .
$$

Let

(see Fig. 6.3). Then

$$
K \equiv A-\operatorname{cl}(\Gamma) \quad \text { and } \quad J \equiv F^{q}(K)
$$

$$
\operatorname{cl}(J) \subset K .
$$

Let $\hat{A}$ and $\hat{A}_{k}$ be as in the proof of Lemma 3 . Let $\hat{K}_{k}$ and $\hat{J}_{k}$ be the components of the lifts of $K$ and $J$ such that

$$
\hat{K}_{k} \subset \hat{A}_{k} \text { and } \hat{J}_{k} \subset \hat{A}_{k} .
$$

Since $\operatorname{cl}\left(\hat{J}_{k}\right) \subset \hat{K}_{k}$, since $\operatorname{cl}\left(\hat{J}_{k}\right)$ is compact, and since $\hat{A}-\hat{K}_{k}$ is closed,

$$
\Delta \equiv d\left(\operatorname{cl}\left(\hat{J}_{k}\right), \hat{A}-\hat{K}_{k}\right)>0 .
$$


Note that $\Delta$ is independent of $k$. Let $\gamma_{n}(\varepsilon)$ be given by Lemma 6 , and choose $\varepsilon$ so small that $\gamma_{q}(\varepsilon)<\Delta$.

Now let $\left\{z_{i}\right\}$ be an $\varepsilon$-pseudo-orbit in $A$. If $z_{i} \in \Gamma$ for all $i$, then the orbit of $y$ is a $\delta$ shadow of $\left\{z_{i}\right\}$ and $\varrho\left(\left\{z_{i}\right\}\right)=\varrho(y)=p / q$. Therefore we may assume that $z_{m} \in \operatorname{cl}(K)$ for some $m$.

Choose the lift $\left\{\hat{z}_{i}\right\}$ so that $\hat{z}_{m} \subset \hat{K}_{0}$. Since $F^{q}\left(z_{m}\right) \in \operatorname{cl}(J)$, we must have

$$
\hat{F}^{q}\left(\hat{z}_{m}\right) \in \operatorname{cl}\left(\hat{J}_{p}\right) .
$$

By Lemma $6, d\left(\hat{F}^{q}\left(\hat{z}_{m}\right), \hat{z}_{m+q}\right) \leqq \gamma_{q}(\varepsilon)<\Delta$, so

$$
\hat{z}_{m+q} \in \hat{K}_{p} .
$$

By induction we can show that

$$
\hat{z}_{m+n q} \in \hat{K}_{n p} \subset \hat{A}_{n p} \text { for all } n .
$$

Lemma 5 implies that $\varrho\left(\left\{z_{i}\right\}\right)=p / q$, and the proof is complete.

We now complete the proof of Theorem I by showing that every orbit in $B$ eventually becomes the shadow of some pseudo-orbit in $A$.

Lemma 8. Let $x \in B$. Given $\varepsilon>0$ and $\delta>0$, there exists a positive integer $N$ and an $\varepsilon$ pseudo-orbit $\left\{z_{i}\right\} \subset A$ such that

$$
d\left(z_{n}, F^{n}(x)\right)<\delta \quad \text { for all } n \geqq N .
$$

Proof. Choose $0<\eta \leqq \min (\varepsilon / 2, \delta)$ so that

$$
d\left(\xi_{1}, \xi_{2}\right)<\eta \text { implies } d\left(F\left(\xi_{1}\right), F\left(\xi_{2}\right)\right)<\varepsilon / 2 \text {. }
$$

Let

$$
H=\{x \in B: d(x, A)<\eta\} .
$$

Choose $N$ so large that $F^{N}(B) \subset H$. Then $d\left(F^{n}(x), A\right)<\eta$ for all $n \geqq N$. Now, for each $n \geqq N$, choose $z_{n} \in A$ such that

$$
d\left(F^{n}(x), z_{n}\right)<\eta \leqq \delta
$$

Then

$$
\begin{aligned}
d\left(F\left(z_{n}\right), z_{n+1}\right) & \leqq d\left(F\left(z_{n}\right), F^{n+1}(x)\right)+d\left(F^{n+1}(x), z_{n+1}\right) \\
& \leqq \varepsilon / 2+\eta \leqq \varepsilon,
\end{aligned}
$$

so $\left\{z_{i}\right\}$ is an $\varepsilon$-pseudo-orbit, and the proof is complete.

\section{Homoclinic Orbits}

Theorem I gives us sufficient conditions for the rotation number to be unique. We now state and prove Theorem II, which gives sufficient conditions for the existence of an interval of rotation numbers. We shall return at the end of this section to the relation between Theorems I and II and to their application to the delayed regulation map. We first need some further definitions. 
Definition. Let $Y \subset B$ be a periodic orbit. A point $x \in B$ is said to be homoclinic to $Y$ if $x \in W^{s}(Y, F) \cap W^{u}(Y, F)-Y$. It is said to be a nondegenerate homoclinic point if $W^{s}$ and $W^{u}$ intersect transversely at $x$. If they intersect tangentially at $x$, then $x$ is said to be a point of homoclinic tangency.

Definition. Let $Y$ and $Y^{\prime}$ be different periodic orbits. A point $x$ is said to be heteroclinic from $Y$ to $Y^{\prime}$ if $x \in W^{u}(Y, F) \cap W^{s}\left(Y^{\prime}, F\right)$. If either $Y$ or $Y^{\prime}$ is a fixed point, then the same definition applies.

Theorem II. Let $y \in A$ be a periodic point of period $q$ and rotation number $p / q$, where $p$ and $q$ are relatively prime. Suppose that $W^{u}\left(y, F^{q}\right)$ intersects $W^{s}\left(F^{k}(y), F^{q}\right)$ transversely for some $0<k<q$. Then there exists a nontrivial interval I containing $p / q$ such that, for every $\alpha \in I$, there is a $z \in A$ with $\varrho(z)=\alpha$. Furthermore, there exist points in $A$ for which the rotation number does not exist.

Note that these hypotheses imply that there is a nondegenerate homoclinic point to the periodic orbit of $y$. Indeed, they also imply something about the structure of the homoclinic orbit. Each iterate of $y$ is a fixed point for $F^{q}$. The homoclinic point must be heteroclinic between two different iterates of $y$. One can easily construct counterexamples to Theorem II for which there is a nondegenerate homoclinic point which is not heteroclinic between two different iterates of $y$.

Note also that the existence of a nondegenerate homoclinic point implies the existence of a Smale horseshoe and hence of an invariant Cantor set on which the map is conjugate to a Bernoulli shift [27]. However, without further construction, one cannot conclude the existence of an interval of rotation numbers. Instead of the Smale construction, we use the machinery of pseudo-orbits and shadows defined in Sect. 6. We need the following standard definition [3].

Definition. An invariant set $\Lambda \subset R^{2}$ is said to have the shadowing property if, for each $\delta>0$, there is an $\varepsilon>0$ such that every $\varepsilon$-pseudo-orbit in $\Lambda$ has a $\delta$-shadow in $R^{2}$.

We use the fact that the closure of a nondegenerate homoclinic orbit has the shadowing property [3-5]. We need only construct pseudo-orbits with the desired rotation number, since we then are assured that true orbits with the same rotation numbers exist.

Proof of Theorem II. Let $y^{\prime}=F^{k}(y)$ and let $x$ be a point of transverse intersection of $W^{u}\left(y, F^{q}\right)$ and $W^{s}\left(y^{\prime}, F^{q}\right)$ (see Fig. 7.1). Let $\Lambda$ be the closure of the orbit of $x$. Choose $\delta \in(0, \pi)$ satisfying

$$
\delta<d\left(\Lambda, R^{2}-B\right)
$$

Use the shadowing property of $\Lambda$ to find an $\varepsilon \in(0, \pi)$ such that every $\varepsilon$-pseudo-orbit in $A$ has a $\delta$-shadow in $R^{2}$. Condition (7.1) insures that the $\delta$-shadow will be in $B$ and hence in $A$. The conditions $\varepsilon<\pi$ and $\delta<\pi$ insure that the pseudo-orbit and its shadow share rotation numbers.

Choose $x_{0} \in \Lambda$ and a positive integer $N$ such that

$$
d\left(x_{0}, y^{\prime}\right)<\varepsilon / 2 \text { and } d\left(F^{N q-k}\left(x_{0}\right), y^{\prime}\right)<\varepsilon / 2
$$




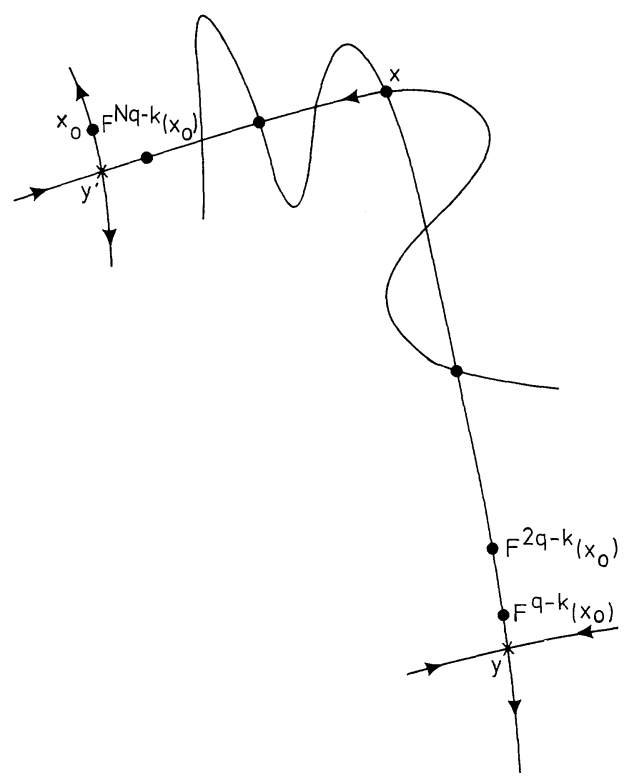

Fig. 7.1. Schematic drawing of part of a homoclinic orbit: $x \in W^{u}\left(y ; F^{q}\right) \cap W^{s}\left(y^{\prime} ; F^{q}\right)$. The point $x_{0}$ and its iterates are used in the construction of a pseudo-orbit with rotation number different from that of the periodic saddle $y$

(see Fig. 7.1). Let $s=N q-k$, and let

$$
\begin{gathered}
H=\left(x_{0}, F\left(x_{0}\right), F^{2}\left(x_{0}\right), \ldots, F^{s-1}\left(x_{0}\right)\right), \\
Y=\left(y^{\prime}, F\left(y^{\prime}\right), F^{2}\left(y^{\prime}\right), \ldots, F^{q-1}\left(y^{\prime}\right)\right) .
\end{gathered}
$$

We denote the juxtaposition of sequences with a "+", i.e.,

$$
H+Y=\left(x_{0}, F\left(x_{0}\right), \ldots, F^{s-1}\left(x_{0}\right), y^{\prime}, F\left(y^{\prime}\right), \ldots, F^{q-1}\left(y^{\prime}\right)\right),
$$

with similar definitions for $Y+H, Y+Y$, and $H+H$. Also, we write

$$
2 H=H+H, 3 H=H+2 H, \text { etc. }
$$

Condition (7.2) insures that any bi-infinite juxtaposition of $H$ and $Y$ is an $\varepsilon$ pseudo-orbit.

First consider the pseudo-orbit $\Psi_{0}=\ldots+Y+Y+Y+\ldots$ This pseudo-orbit is in fact a real orbit, the periodic orbit of $y$, and has rotation number $p / q$.

Next consider the pseudo-orbit $\Psi_{1}=\ldots+H+H+H+\ldots$ Let $r$ be the number of times the sequence $H$ traverses the annulus. That is, $r$ is the unique integer satisfying

$$
\left|\arg \hat{F}^{s}\left(\hat{x}_{0}\right)-\arg \hat{x}_{0}-2 \pi r\right|<\varepsilon .
$$

Then the rotation number of $\Psi_{1}$ is $r / s$. Since $p$ and $q$ are relatively prime, and since $q$ does not divide $s$, we have

$$
r / s \neq p / q .
$$


We take I to be the nontrivial interval between $p / q$ and $r / s$.

Now let $a \in I$ be rational. We can write

$$
a=\frac{u p+v r}{u q+v s}
$$

for some positive integers $u$ and $v$ [23]. Consider the pseudo-orbit

$$
\Psi_{2}=\ldots+(u Y+v H)+(u Y+v H)+(u Y+v H)+\ldots
$$

The rotation number of $\Psi_{2}$ is $a$, as can be seen by counting the rotations of the pseudo-orbit segment $u Y+v H$.

We now construct orbits with irrational rotation numbers. Let $\alpha \in I$ be irrational and pick a sequence of rationals $a_{i} \in I$ such that $a_{i} \rightarrow \alpha$ as $i \rightarrow \infty$. For each $i$, write

$$
a_{i}=\frac{u_{i} p+v_{i} r}{u_{i} q+v_{i} s}
$$

and consider the pseudo-orbit

$$
\Psi_{3}=\ldots+n_{1}\left(u_{1} Y+v_{1} H\right)+n_{2}\left(u_{2} Y+v_{2} H\right)+n_{3}\left(u_{3} Y+v_{3} H\right)+\ldots,
$$

where $n_{i}$ is chosen so that $u_{i+1} / n_{i} \rightarrow 0$ as $i \rightarrow \infty$. This pseudo-orbit has rotation number $\alpha$.

Finally, we construct a pseudo-orbit whose rotation number does not exist. Let $n_{i}$ be a sequence of positive integers, and let

Also let

$$
\Psi_{4}=\ldots+n_{1} Y+n_{2} H+n_{3} Y+n_{4} H+\ldots
$$

$$
\begin{gathered}
u_{i}= \begin{cases}n_{1}+n_{3}+\ldots+n_{i-1} & \text { if } i \text { is even } \\
n_{1}+n_{3}+\ldots+n_{i} & \text { if } i \text { is odd }\end{cases} \\
v_{i}= \begin{cases}n_{2}+n_{4}+\ldots+n_{i} & \text { if } i \text { is even } \\
n_{2}+n_{4}+\ldots+n_{i-1} & \text { if } i \text { is odd }\end{cases} \\
a_{i}=u_{i} p+v_{i} r, \\
b_{i}=u_{i} q+v_{i} s,
\end{gathered}
$$

and

$$
\varrho_{i}=a_{i} / b_{i}
$$

If $\varrho\left(\Psi_{4}\right)$ exists, then it is equal to $\lim _{i \rightarrow \infty} \varrho_{i}$. Note that

$$
\varrho_{i}= \begin{cases}\frac{a_{i-1}+n_{i} p}{b_{i-1}+n_{i} q} & \text { if } i \text { is odd, } \\ \frac{a_{i-1}+n_{i} r}{b_{i-1}+n_{i} s} & \text { if } i \text { is even } .\end{cases}
$$

If we choose $n_{i}$ so that $\left(n_{1}+n_{2}+\ldots+n_{i-1}\right) / n_{i} \rightarrow 0$ as $i \rightarrow \infty$, then

$$
\varrho_{2 j} \rightarrow r / s \text { but } \varrho_{2 j+1} \rightarrow p / q \text { as } j \rightarrow \infty \text {. }
$$




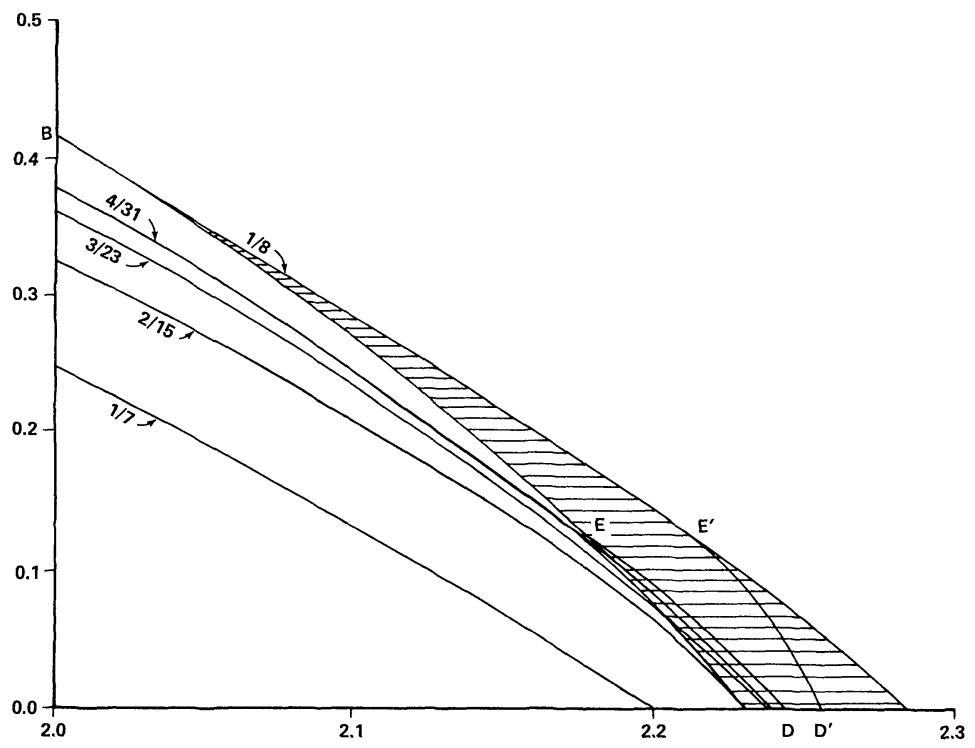

Fig. 7.2. The shaded region is the resonance horn for rotation number $1 / 8$. The curves labeled $1 / 7, \ldots, 4 / 31$ are the upper boundaries of the corresponding resonance horns. Homoclinic tangencies for the 8 period saddle occur for parameter values on the curves $E D$ and $E^{\prime} D^{\prime}$

Thus the limit of $\varrho_{i}$ does not exist, and the pseudo-orbit $\Psi_{4}$ does not have a rotation number. The proof of Theorem II is complete.

Before proceeding, we would like to make two observations about the proof.

The general theory of shadowing tells us that the shadow of one of the pseudoorbits constructed above is periodic if and only if the pseudo-orbit is periodic. Since $\Psi_{2}$ is periodic, it follows that the shadow of $\Psi_{2}$ is a periodic orbit with rotation number $a$. Therefore we have actually constructed a periodic orbit for each rational rotation number in $I$.

One should note also that our definition of rotation number uses the limit as $n \rightarrow+\infty$. Let us call this limit $\varrho^{+}(x)$. We could also use the limit as $n \rightarrow-\infty$, giving us the asymptotic rotation number as we proceed backward in time. Let us call this latter limit $\varrho^{-}(x)$. The above constructions can be modified to produce an orbit with the following property. For any $\alpha^{+} \in I$ and $\alpha^{-} \in I$ there exists a point $x \in B$ such that

$$
\varrho^{+}(x)=\alpha^{+} \text {and } \varrho^{-}(x)=\alpha^{-} .
$$

We now turn to the question of how the hypotheses of Theorem I can fail. We always assume that we take parameter values inside the $p / q$ resonance horn, so hypothesis (a) always holds. We first point out that it is possible for (c) to fail while (b) is valid, i.e. for both branches of $W^{s}\left(y, F^{q}\right)$ to lie on the same side of $A$. This possibility is illustrated in Fig. 9.3g and is discussed in Sect. 9. However, if we start at the tip of the resonance horn and vary the parameters continuously, hypothesis (c) cannot fail unless (b) fails first. Although hypothesis (b) can be violated in many ways, we have witnessed only one in our computer explorations of the resonance horns: the formation via a homoclinic tangency of a homoclinic point satisfying 


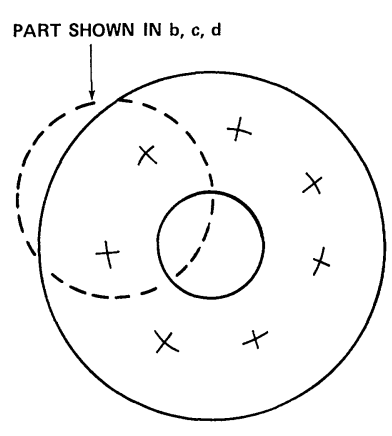

(a)

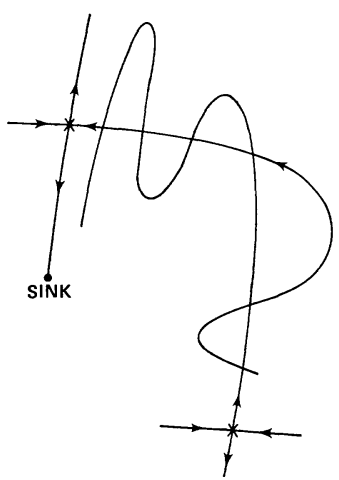

(c)
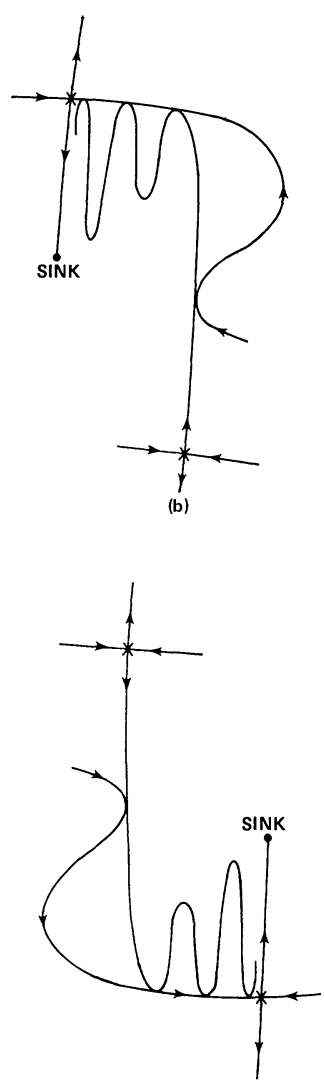

(d)

Fig. 7.3. Schematic drawing of the transition from $\mathbf{b}$ homoclinic tangency to $\mathbf{c}$ homoclinic crossing to d homoclinic tangency on the opposite side

the hypotheses of Theorem II. The set of parameter values for which this tangency occurs usually defines an arc in the two-dimensional parameter space.

For the two-parameter family $F_{(a, b)}$ discussed in Sect. 5, one of these arcs is represented by $E D$ in Fig. 7.2 for the 1/8 resonance horn. Although we have computed the boundaries of the resonance horns quite accurately, the arc $E D$ is sketched using only a few data points. Along this arc we have the homoclinic tangency shown in Fig. 7.3b. Below this arc there is a homoclinic point as shown in Fig. 7.3c. Above this arc, there is no homoclinic point of the type shown.

There is an analogous arc on the other side of the resonance horn, represented by $\operatorname{arc} E^{\prime} D^{\prime}$ in Fig. 7.2. It is the arc where the other side of the unstable manifold of the saddle is involved in the homoclinic tangency, as illustrated in Fig. 7.3d.

In addition to the $1 / 8$ resonance horn, the right hand boundaries of the resonance horns 1/7, 2/15,3/23, and 4/31 are shown in Fig. 7.2. All the numerical evidence we have is consistent with the proposition that the arc $E D$ is the limit of these right hand boundaries. Our evidence also shows that $1 / 8$ is the only rotation 


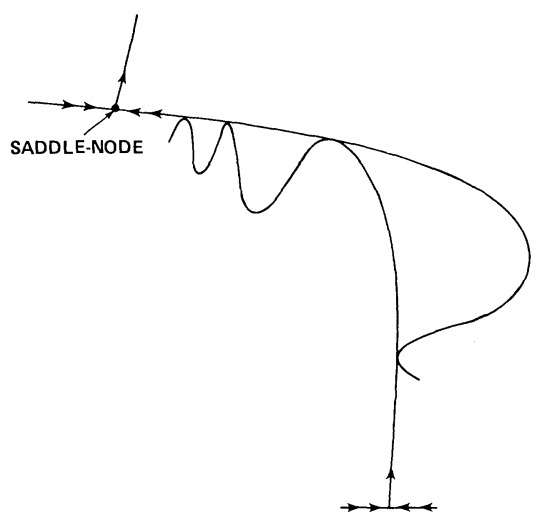

Fig. 7.4. Schematic drawing of a homoclinic tangency between saddle-nodes corresponding to the point $E$ in Fig. 7.2

number present in the region bounded by $D E B E^{\prime} D^{\prime} D$, while other rotation numbers are present outside of this region in the resonance horn.

The reader should compare Fig. 7.2 with the rotation number graph in Fig. 3.4. The points $D$ and $D^{\prime}$ are labeled in both figures.

Homoclinic tangencies were previously studied by Newhouse [20]. His work indicates that we can expect an infinite number of sinks for certain parameter values near the homoclinic tangency. Although all the periodic orbits constructed using pseudo-orbits are saddles, they probably arise as saddle-sink pairs bifurcating from saddle-nodes. Since these saddle-nodes occur along the boundaries of the resonance horns, and since some of these boundaries accumulate along the arc $E D$ in Fig. 7.2, one might expect an infinite number of sinks for some parameter values near this arc. The relation between this structure and Newhouse's theory remains to be studied.

The arc of homoclinic tangency intersects the boundary of the resonance horn at a point marked $E$ in Fig. 7.2. Recall that the saddle and sink come together to form a saddle-node when the parameters are on the boundary of the horn. At the point $E$ the unstable manifold of the saddle-node is tangent to its own strong stable manifold, as shown in Fig. 7.4. We shall return to this point in Sect. 8.

\section{Loss of Smoothness of the Invariant Circle}

After a homoclinic point has developed, the attractor $A$ for the map $f_{\mu}$ no longer can be even topologically a circle. However, for parameter values inside the resonance horn near the unit circle, the attractor is known to be a smooth circle. We now address the question of how this circle can lose its smoothness as the parameters change.

The first answer which comes to mind concerns the relative strengths of the eigenvalues of the periodic sink. Since the invariant circle is the union of the unstable manifold of the periodic saddle with the periodic sink, and since the unstable manifold is as smooth as the map itself, the smoothness of the invariant 
circle is determined by the way the two branches of the unstable manifold join together at the sink.

For parameter values near the Hopf bifurcation or near the boundaries of the horn, the sink will have one eigenvalue close to 1 and the other eigenvalue strictly less than 1 . The eigenvector corresponding to the eigenvalue close to 1 is tangent to the invariant circle and represents the weak, or slow, rate of approach. The other eigenvector is transverse to the circle and represents the strong, or fast, rate of approach. Recall that there is a distinguished strong stable manifold of orbits asymptotic to the sink and tangent to the strong eigenvector. This strong stable manifold is indicated by double arrows in Fig. 2.1.

Now suppose that one branch of the unstable manifold comes into the sink on one side of the strong stable manifold, while the other branch comes into it on the other side. This assumption is valid in a set which includes parameter values near the unit circle.

To illustrate the effect of the sink on the smoothness of the invariant circle, we consider the following model. Suppose that we have a map $f: R^{2} \rightarrow R^{2}$ with a sink at the origin and a saddle elsewhere. Suppose that, in a neighborhood of the origin, $f$ can be written

$$
(x, y) \rightarrow(\alpha x, \beta y)
$$

where $0<\beta<\alpha<1$. Note that the $y$-axis is the strong stable manifold. Assume that the unstable manifold of the saddle can be written as the graph of a function $y=\psi(x)$. Since the unstable manifold is as smooth as $f$, we can assume that $\psi$ is real analytic except possibly at the origin. A standard argument shows that, if $\beta<\alpha^{k}, \psi$ is a $C^{k}$ function at the origin [7].

To see that $\psi$ cannot be expected to be $C^{k}$ at the origin when $\beta>\alpha^{k}$, consider the curve

$$
y=|x|^{r} \text {, where } r=\frac{\log \beta}{\log \alpha} .
$$

This curve is invariant under the map (8.1) and is real analytic everywhere except at $x=0$, where it fails to be $C^{k}$. Here we are also assuming that $r$ is not an integer.

Using the above model as a heuristic guide, we formulate the following conjecture about the invariant circle for the map $f_{\mu}$. Let $\alpha$ and $\beta$ be the eigenvalues of the sink, with $\beta<\alpha$. If $\beta<\alpha^{k}$, then we expect the invariant circle to be $C^{k}$. If $\beta>\alpha^{k}$, then we do not expect the circle to be $C^{k}$.

We can look for the parameter values inside the resonance horn where $\beta=\alpha^{k}$. In general, we expect this equation to define a curve for each $k$. Since $\alpha \rightarrow 1$ as the parameters approach the boundaries of the resonance horn, we expect these curves to accumulate against the boundaries as $k \rightarrow \infty$, as shown in Fig. 8.1. As we move the parameters into the horn, we expect the invariant circle to lose smoothness.

A particularly important case occurs when the circle fails to be even $C^{1}$. Following the above considerations, we expect this failure to occur when $\alpha=\beta$, so that the sink develops a multiple eigenvalue. We then expect the eigenvalues to become complex, so that the invariant circle develops an infinite spiral, as shown in Fig. 8.2. We have indeed observed such spirals for the maps $F_{a}$ and $F_{(a, b)}$. 


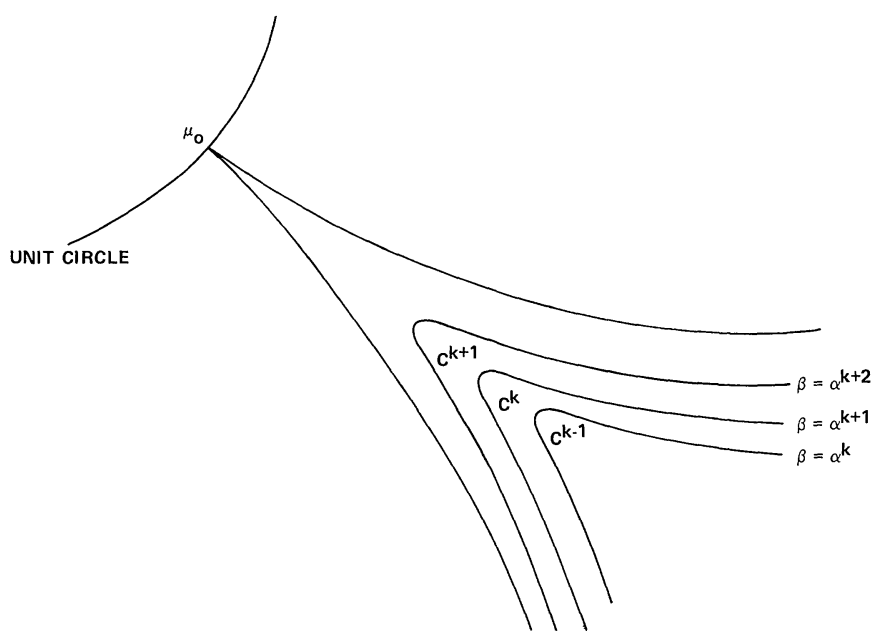

Fig. 8.1. Sketch of a typical resonance horn in the complex $\mu$-plane. The indicated functional relationship between the eigenvalues $\alpha, \beta$ of the sink holds on curves shown inside the horn. We can expect the invariant circle to be no more than $C^{k+1}$ in the subregion of the horn below the curve labeled $\beta=\alpha^{k+2}$

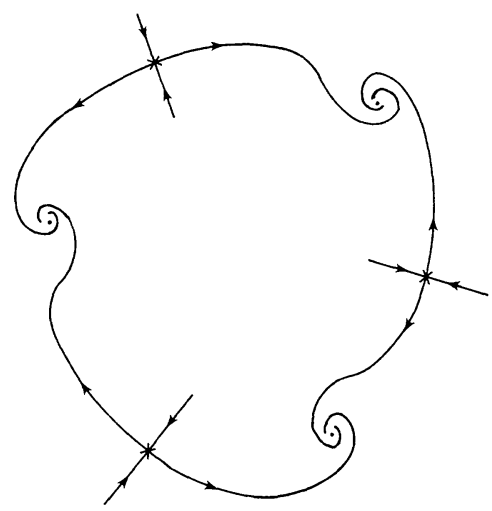

Fig. 8.2. Schematic drawing of a saddle-sink pair of periodic orbits when the sink has complex eigenvalues

However, there is another way in which the circle can fail to be $C^{1}$. To see this alternate way, we again appeal to the map $f$ discussed above. This time we do not assume that the unstable manifold can be written as the graph of a function $y=\psi(x)$, but instead assume that it contains a point with a vertical tangent, as shown in Fig. 8.3a. Since the vertical tangent remains vertical under iteration of the map, there will be points arbitrarily close to the origin with vertical tangents. Since there will also be points arbitrarily close to the origin with nearly horizontal tangents, the unstable manifold extended to the origin is not $C^{1}$.

To be more precise about the relation between the above model and the invariant circle after Hopf bifurcation, we must first discuss a certain splitting of 

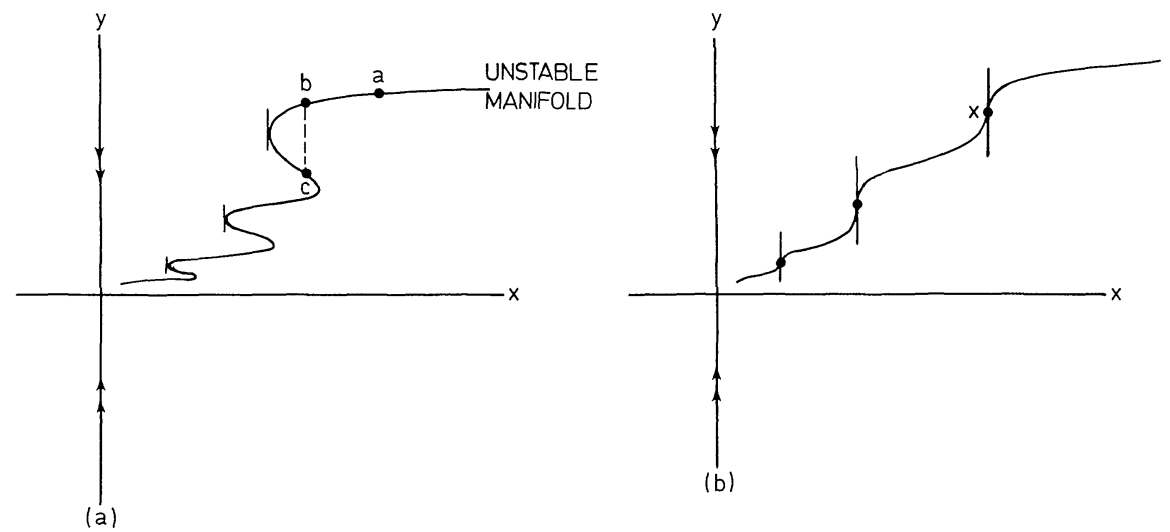

Fig. 8.3a and b. Schematic drawing of the unstable manifold from a distant saddle point approaching a sink for the model (8.1). Because of recurrent vertical and horizontal tangents the invariant circle is not $C^{1}$ in any neighborhood of the sink. The distance between the successive images of the points labeled $a$ and $b$ tend to zero at the slow asymptotic rate $\alpha^{n}$ while for the points $b$ and $c$ the corresponding distances tend to zero at the fast rate $\beta^{n}$. b The transition state which separates the behavior described in a from the case in which the invariant circle is $C^{1}$

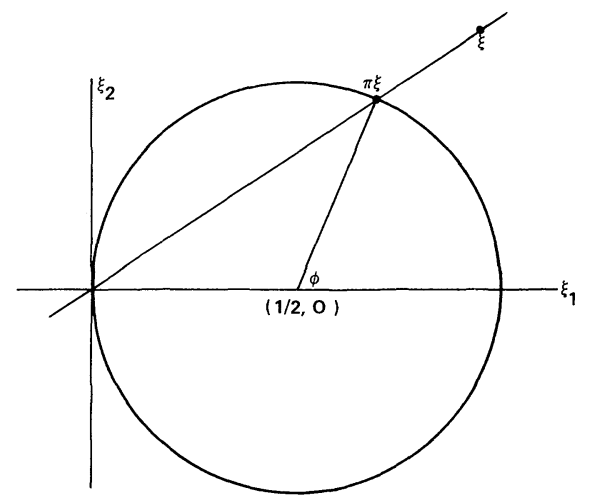

Fig. 8.4. Projection of the line bundle over a point in $\mathbb{R}^{2}$ onto a circle with center $(1 / 2,0)$ and radius $1 / 2$. Each line through the origin corresponds to a unique point on the circle as shown

the tangent bundle. Consider again the map $F: B \rightarrow B$, where $B$ is an annular attractor block. For $x \in B$, we have the derivative, or Jacobian, map

$$
D F(x): R^{2} \rightarrow R^{2},
$$

which gives us the map on the tangent bundle

$$
T F: B \times R^{2} \rightarrow B \times R^{2}:(x, \xi) \rightarrow(F(x), D F(x) \xi) .
$$

Now consider the real projective circle $P^{1}$, that is, the space of lines through the origin in $R^{2}$. We identify $P^{1}$ with a circle as follows:

$$
P^{1} \cong\left\{\left(\xi_{1}, \xi_{2}\right) \in R^{2}: \xi_{1}^{2}+\xi_{2}^{2}=\xi_{1}\right\} .
$$

Every line through the origin corresponds to exactly one point on the circle, as shown in Fig. 8.4. The vertical line corresponds to the origin, while every other line 
corresponds to the non-trivial point of intersection of the line with the circle.

Define the projection

$$
\pi: R^{2}-\{0\} \rightarrow P^{1}:\left(\xi_{1}, \xi_{2}\right) \rightarrow \frac{\xi_{1}}{\xi_{1}^{2}+\xi_{2}^{2}}\left(\xi_{1}, \xi_{2}\right) .
$$

Every non-singular linear map $L$ induces a map $\pi L: P^{1} \rightarrow P^{1}$ which makes the following diagram commute:

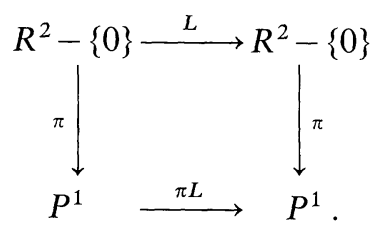

This map can be written:

$$
\pi L(\xi)=\left\{\begin{array}{lll}
\pi(L \xi) & \text { if } & \xi \neq(0,0) \\
\pi(L(0,1)) & \text { if } & \xi=(0,0)
\end{array}\right.
$$

The tangent map $T F$ induces a map on the projective bundle:

$$
\pi T F: B \times P^{1} \rightarrow B \times P^{1}:(x, \xi) \rightarrow(F(x), \pi D F(x)(\xi)) .
$$

We say that the tangent bundle splits if there exist two continuous functions $\sigma_{1}$, $\sigma_{2}: B \rightarrow P^{1}$ such that (1) the graphs of $\sigma_{1}$ and $\sigma_{2}$ are invariant under $\pi T F$, i.e.,

$$
\pi T F\left(x, \sigma_{j}(x)\right)=\left(F(x), \sigma_{j}(F(x))\right) \text { for all } x \in B, j=1,2,
$$

and (2) for all $(x, \xi) \in B \times P^{1}$ such that $\xi \neq \sigma_{2}(x)$,

$$
d\left((\pi T F)^{n}(x, \xi), \operatorname{graph}\left(\sigma_{1}\right)\right) \rightarrow 0 \quad \text { as } n \rightarrow \infty .
$$

The second condition says that, except for those vectors which lie in the graph of $\sigma_{2}$, all tangent vectors are attracted to the direction given by $\sigma_{1}$.

Now assume that the attractor associated with $B$ is a smooth circle in resonance with rotation number $p / q$. Let $y \in B$ be one of the saddles and $v \in B$ be one of the sinks. For each of these points in $B$, there are two periodic points in the projective circle bundle, one for each eigenspace. Let $\mu$ and $\nu$ be the eigenvalues for $y$, with corresponding eigenvectors $\eta_{1}$ and $\eta_{2}$. Assume that $0<v<1<\mu$, and let $y_{1}=\left(y, \pi \eta_{1}\right) \in B \times P^{1}$ and $y_{2}=\left(y, \pi \eta_{2}\right) \in B \times P^{1}$. Note that $\eta_{1}$ is the unstable direction for the saddle point $y$ and hence is tangent to the invariant circle at $y$, while $\eta_{2}$ is the stable direction and is transverse to the circle. Let $\alpha$ and $\beta$ be the eigenvalues for $v$, with corresponding eigenvectors $\chi_{1}$ and $\chi_{2}$. Assume that $0<\beta<\alpha<1$, and let $v_{1}=\left(v, \pi \chi_{1}\right) \in B \times P^{1}$ and $v_{2}=\left(v, \pi \chi_{2}\right) \in B \times P^{1}$. Note that $\alpha$ is the weak eigenvalue and hence that $\chi_{1}$ is tangent to the invariant circle.

For the $q^{\text {th }}$ iterate of the map $\pi T F$ on $B \times P^{1}$, the point $v_{1}$ has eigenvalues $\alpha<1$, $\beta<1$, and $\beta / \alpha<1$, while the point $v_{2}$ has eigenvalues $\alpha<1, \beta<1$, and $\alpha / \beta>1$. Hence $v_{1}$ is a sink, while $v_{2}$ is a saddle with two contracting directions and one expanding direction, as shown in Fig. 8.5. These two contracting directions are tangent to $B$ in $B \times P^{1}$, while the expanding direction is normal to $B$. Therefore $v_{2}$ 


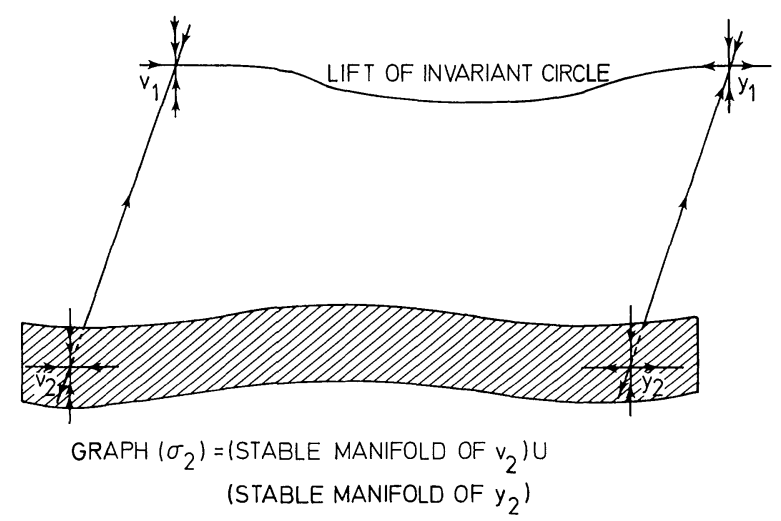

Fig. 8.5. The projective bundle over the annulus showing the lift of the invariant circle, the saddle-sink pair on the invariant circle, and the stable manifold of the strong direction of the sink

has a local stable manifold which is the graph of a smooth function $\sigma_{v}: U \rightarrow P^{1}$, where $U$ is a neighborhood of $v$ in $B$. For $(x, \xi) \in U \times P^{1}$, either $\pi T F^{q n}(x, \xi) \rightarrow v_{1}$ as $n \rightarrow \infty$ or $\pi T F^{q n}(x, \xi) \rightarrow v_{2}$ as $n \rightarrow \infty$, in which case $\xi=\sigma_{v}(x)$. Thus, most tangent vectors over $x \in U$ tend to the weak eigenspace at $v$. However, for each $x \in U$, there is a unique tangent vector $\sigma_{v}(x)$ which tends to the strong eigenspace at $v$.

Now consider the points $y_{1}$ and $y_{2}$ in $B \times P^{1}$. For the $q^{\text {th }}$ iterate of the map $\pi T F$, the point $y_{1}$ has eigenvalues $\mu>1, v<1$, and $v / \mu<1$, while the point $y_{2}$ has eigenvalues $\mu>1, v<1$, and $\mu / v>1$. Hence $y_{1}$ has two contracting directions and one expanding direction, while $y_{2}$ has one contracting direction and two expanding directions. The unstable manifold $W^{u}\left(y_{1}, \pi T F^{q}\right)$ is one dimensional and is the lift to $B \times P^{1}$ of the unstable manifold $W^{u}\left(y, F^{q}\right)$ of $y$ in $B$. That is, $(x, \xi) \in W^{u}\left(y_{1}, \pi T F^{q}\right)$ if and only if $x \in W^{u}\left(y, F^{q}\right)$ and $\xi$ is tangent to $W^{u}\left(y, F^{q}\right)$ at $x$. The stable manifold $W^{s}\left(y_{2}, \pi T F^{q}\right)$ of $y_{2}$ is one dimensional and is the graph of a smooth function $\sigma_{y}: W^{s}\left(y, F^{q}\right) \rightarrow P^{1}$. For $(x, \xi) \in W^{s}\left(y, F^{q}\right) \times P^{1}$, either $\pi T F^{q n}(x, \xi) \rightarrow y_{1}$ as $n \rightarrow \infty$, or $\pi T F^{q n}(x, \xi) \rightarrow y_{2}$ as $n \rightarrow \infty$, in which case $\xi=\sigma_{y}(x)$.

Now let $V, V_{1}, V_{2}, Y, Y_{1}$, and $Y_{2}$ be the orbits of $v, v_{1}, v_{2}, y, y_{1}$, and $y_{2}$, respectively. We can extend $U$ and $\sigma_{v}$ so that $U$ is a neighborhood of $V$ and $\operatorname{graph}\left(\sigma_{v}\right)$ is the stable manifold of $V_{2}$. We can also extend $\sigma_{y}$ so that $\operatorname{graph}\left(\sigma_{y}\right)$ is the stable manifold of $Y_{2}$. If the tangent bundle splits, then $U$ can be extended so that $U \cup W^{s}(Y, F)=B$ and so that

$$
\sigma_{2}(x)=\left\{\begin{array}{lll}
\sigma_{v}(x), & \text { if } & x \in U \\
\sigma_{y}(x), & \text { if } & x \in W^{s}(Y, F)
\end{array}\right.
$$

is a continuous function.

We now turn to the function $\sigma_{1}$, which is not unique but is determined by the saddle point $y_{1}$. Since the eigenvalues of $y_{1}$ satisfy $v / \mu<v<1<\mu$, there is a nonunique invariant manifold $W$ associated with eigenvalues $v$ and $\mu$ and tangent to $B \times\{0\}$. Furthermore, $W^{u}\left(y_{1}, \pi T F^{q}\right) \subset W$, and $W$ matches up continuously with the one dimensional strong stable manifold at $v_{1}$. Therefore, the iterates of $W$ and this strong stable manifold form the graph of some function $\sigma_{1}: B \rightarrow P^{1}$. The splitting is now defined by $\sigma_{1}$ and $\sigma_{2}$. 


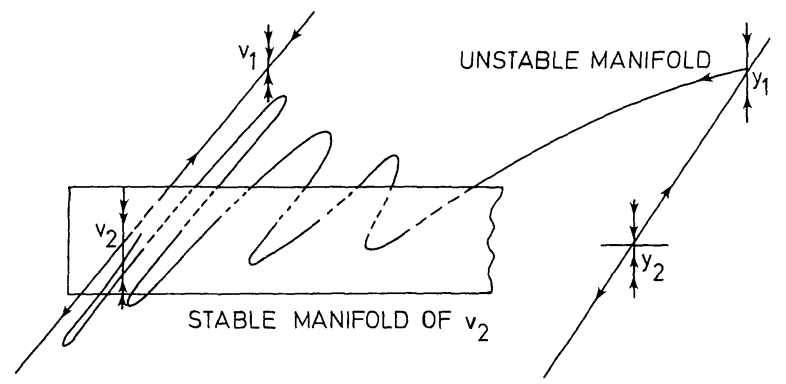

Fig. 8.6. Schematic drawing of a heteroclinic crossing of invariant manifolds in the projective bundle. This corresponds to the structure of the invariant circle shown in Fig. 8.3a

The structure just defined is illustrated in Fig. 8.5. The lift of the invariant circle to $B \times P^{1}$ is the unstable manifold of $y_{1}$, together with the point $v_{1}$. The graph of $\sigma_{2}$ is the stable manifold of $v_{2}$, together with the stable manifold of $y_{2}$. As shown in the figure, the unstable manifold of $y_{1}$ does not intersect the stable manifold of $v_{2}$, and the invariant circle is $C^{1}$.

We have already discussed how the relative strengths of the eigenvalues $\alpha$ and $\beta$ can affect the smoothness of the invariant circle. It is also possible for the invariant circle to cease to be $C^{1}$ by the development of a type of heteroclinic point in $B \times P^{1}$. If the unstable manifold of $y_{1}$ intersects the stable manifold of $v_{2}$, then this unstable manifold will have as limit points both $v_{1}$ and $v_{2}$, as shown in Fig. 8.6. Since the second coordinate of this unstable manifold in $B \times P^{1}$ represents the tangent to the invariant circle in $B$, the invariant circle will have points arbitrarily close to $v$ with very different tangent directions, as was illustrated for the model (8.1) in Fig. 8.3.

Note that there can be no splitting of the tangent bundle in this case, since the stable manifold of $v_{2}$ will behave wildly near $y$, having both $y_{1}$ and $y_{2}$ as limit points.

There is another characterization of this behavior. When the splitting is present, the invariant circle has the following property. Any two points between two consecutive saddles on the circle approach each other at the asymptotic rate given by the slow eigenvalue at the sink. When the heteroclinic point is present, there exist distinct points on the circle which approach each other at the asymptotic rate given by the fast eigenvalue at the sink. This phenomenon is best illustrated in Fig. 8.3a. The distance between the iterates of the points labeled $a$ and $b$ approaches zero at the slow asymptotic rate $\alpha^{n}$, whereas the distance between the iterates of $b$ and $c$ approaches zero at the fast asymptotic rate $\beta^{n}$.

The transition between the presence and the absence of this heteroclinic behavior occurs when the unstable manifold of $y_{1}$ becomes tangent to the stable manifold of $v_{2}$. The interpretation of this tangency in terms of the model (8.1) is shown in Fig. 8.3b. A point $x$ where the unstable manifold of $y_{1}$ is tangent to the stable manifold of $v_{2}$ corresponds to a point where the tangent line to the unstable manifold shown in the figure is vertical, but nearby tangent lines have positive slope. 


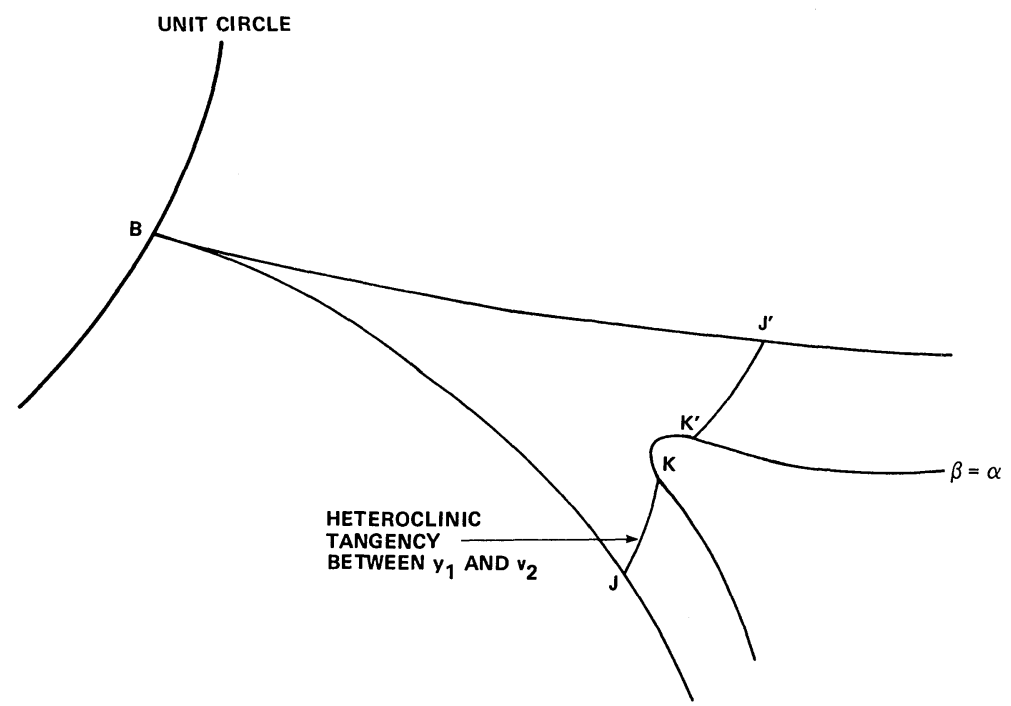

Fig. 8.7. Sketch of a typical resonance horn in the complex $\mu$-plane. The curve labeled $\beta=\alpha$ indicates where the eigenvalues $\alpha, \beta$ of the sink are equal. The curves $J K$ and $J^{\prime} K^{\prime}$ indicate parameter values for which heteroclinic tangencies occur in the projective bundle. Points on $J K$ correspond to the structure of the invariant circle shown in Fig. $8.3 \mathrm{~b}$

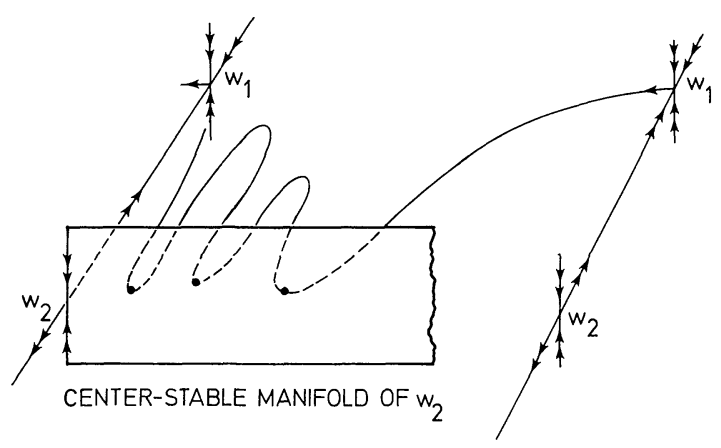

Fig. 8.8. Heteroclinic tangency in the projective bundle over a saddle-node. This corresponds to the point $J$ in Fig. 8.7

The heteroclinic tangency which we have just described can occur regardless of the relative strengths of the eigenvalues at the sink. Computer simulations indicate that the arc of parameter values for which this tangency occurs connects the boundary of the horn with the curve of double eigenvalues, as shown by the arcs $J K$ and $J^{\prime} K^{\prime}$ in Fig. 8.7. In the region bounded by $J K K^{\prime} J^{\prime} B J$, the attractor $A$ is a $C^{1}$ invariant circle. Again, this figure represents computations on the map $F_{(a, b)}$ for parameters inside the $1 / 8$ resonance horn.

The behavior of the map at the point $J$ is illustrated in Fig. 8.8. Since this point is on the boundary of the resonance horn, the sink $v$ and the saddle $y$ have come together to form a saddle-node $w$. The point $w_{1}$ corresponds to the eigenvalue one, while the point $w_{2}$ corresponds to the eigenvalue which is less than one. The point 
$w_{2}$ has a two-dimensional center-stable manifold which is the continuation to $w$ of the stable manifold of $u_{2}$. The point $w_{1}$ has a one-dimensional unstable manifold, which is the lift to $B \times P^{1}$ of the unstable manifold of $w$ in $B$. For the parameter values represented by the point $J$, the unstable manifold of $w_{1}$ is tangent to the center-stable manifold of $w_{2}$.

\section{Other Interactions Involving the Unstable Manifolds}

We now briefly discuss some of the other phenomena occurring inside the resonance horn. In this discussion we refer to Fig. 9.1, which is a sketch of the $1 / 8$ resonance horn for the map $F_{(a, b)}$. The labels in Fig. 9.1 refer to the sketches in Figs. 9.2-9.4, and will be discussed in detail below. These figures are schematic representations of pictures generated by the computer. In drawing them we have exaggerated the essential features for the purposes of illustration. Also, our data are not complete, and Fig. 9.1 represents only our best guess based on computer observations to date.

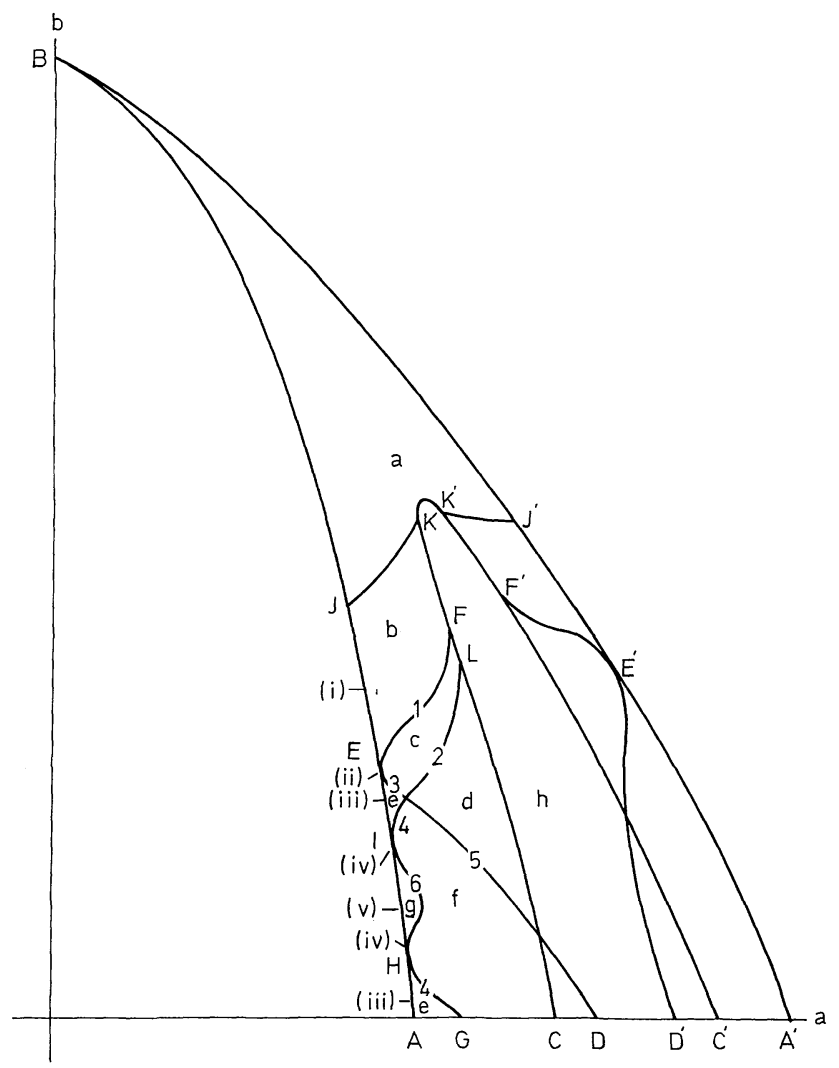

Fig. 9.1. Schematic representation of the structure observed in a typical resonance horn for the twoparameter delayed logistic map. The labels (i)-(v) correspond to the saddle-node configurations shown in Fig. 9.2, the regions $a-h$ correspond to the structures shown in Fig. 9.3, and the curves 1-6 correspond to the various types of tangencies shown in Fig. 9.4 


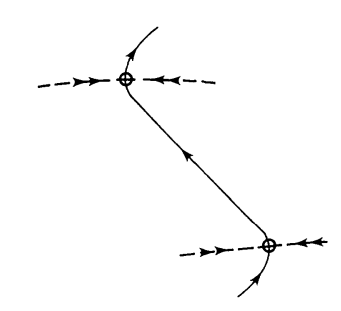

(i) NORMAL

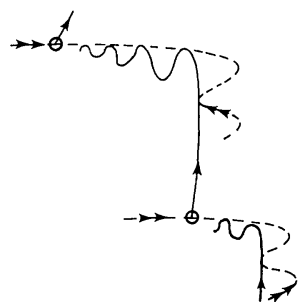

(ii) HOMOCLINIC TANGENCY (I)

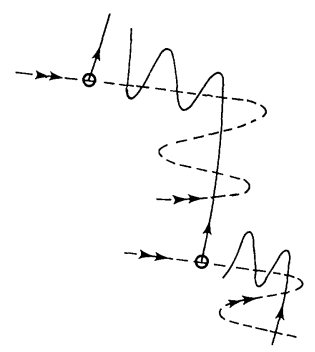

(iii) HOMOCLINIC CROSSING

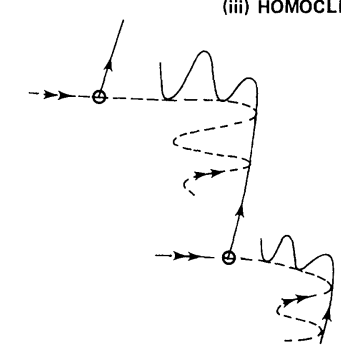

(iv) HOMOCLINIC TANGENCY (II)

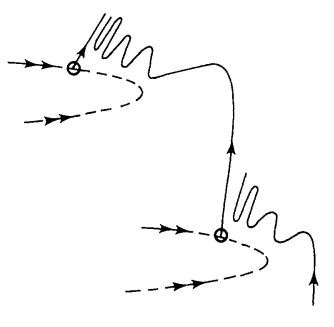

(v) DISCONNECTED

Fig. 9.2. Saddle-nodes

The boundaries of the horn are the curves $B A$ and $B A^{\prime}$, representing those parameter values for which the $1 / 8$ saddle-nodes occur, as discussed in Sect. 4 . The curve $C L F K K^{\prime} F^{\prime} C^{\prime}$ represents those parameters for which the eigenvalues of the sink coincide. Above this curve these eigenvalues are real. Immediately inside this curve these eigenvalues are complex, and the invariant circle has spirals, as shown in Fig. 9.3h. We shall discuss the region inside of this curve in more detail in Sect. 10.

The arcs $J K$ and $J^{\prime} K^{\prime}$ were discussed in Sect. 8. Above the curve $J K K^{\prime} J^{\prime}$ the invariant circle is at least $C^{1}$. The arc $E D$ represents the parameter values for which there is a homoclinic tangency, as discussed in Sect. 7.

At the point $E$, the unstable manifold of the saddle-node is tangent to its own strong stable manifold, as shown in Fig. 9.2(ii). The behavior of the map for parameter values in a neighborhood of the point $E$ is illustrated in the following model:

$$
(x, y) \rightarrow\left(r x, y-\varepsilon+(y-\delta)^{2}\right),
$$

where $r$ is a fixed constant with $0<r<1$, and where $\varepsilon$ and $\delta$ are parameters. This map is exactly the map (4.2), except that the $x$-axis has been translated by $\delta$. Therefore, Fig. 4.2 illustrates the orbit structure of map (9.1). For $\varepsilon>0$, there is a 


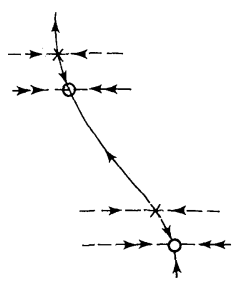

(a) $c^{1}$

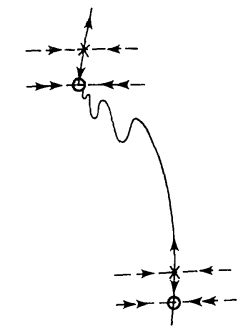

(b) DIFFERENTIABLE, BUT NOT $C^{1}$

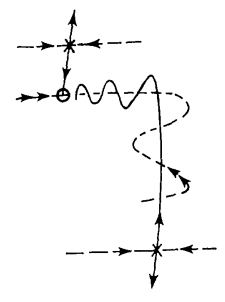

(c) HETEROCLINIC CROSSING

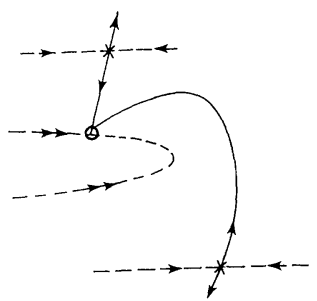

(d) CUSP

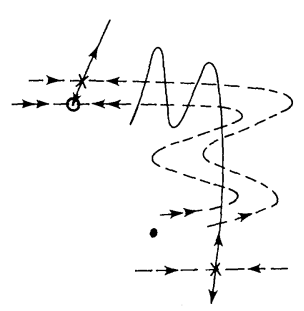

(e) HOMO/HETEROCLINIC

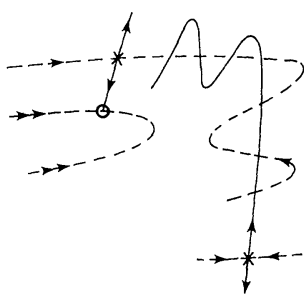

(f) HOMOCLINIC CROSSING

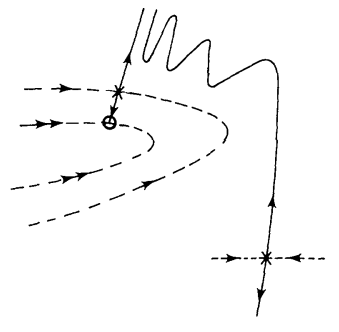

(g) DISCONNECTED

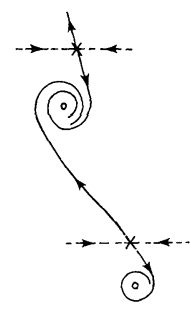

(h) SADDLE-SPIRAL PAIR

Fig. 9.3. Saddle-sink pairs. Saddles are indicated by $\times$ and sinks by 0

saddle point at $(x, y)=(0, \delta+\sqrt{\varepsilon})$ and a sink at $(x, y)=(0, \delta-\sqrt{\varepsilon})$, as shown in Fig. 9.5. As $\varepsilon \rightarrow 0$, these two points come together to form a saddle-node at $(x, y)=(0, \delta)$.

We now assume that (9.1) represents the local structure of the map, but that globally the unstable manifold of the saddle comes back and is tangent to the $x$ axis, as shown in Fig. 9.5. The homoclinic tangency will then occur for parameters along the curve $\delta=-\sqrt{\varepsilon}$, as shown in Fig. 9.6. The curve $\delta=+\sqrt{\varepsilon}$ represents the parameter values for which the unstable manifold of the saddle is tangent to the strong stable manifold of the sink. We shall refer to this latter phenomenon as "heteroclinic tangency."

We consider the map (9.1) as a model for the $q^{\text {th }}$ iterate of $F_{(a, b)}$ near the point $E$. The $\delta$-axis represents the boundary of the resonance horn, with $\delta$ increasing toward the point $B$ of Hopf bifurcation. The parameter $\varepsilon$ is transverse to the 


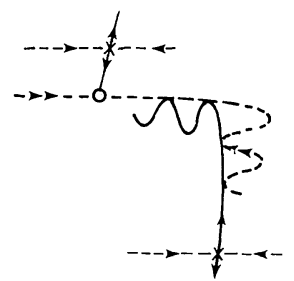

(1) HETEROCLINIC TANGENCY (I)

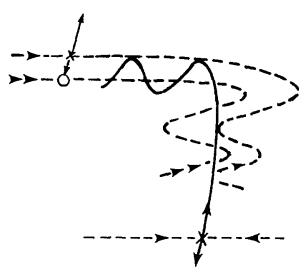

(3) HOMOCLINIC TANGENCY HETEROCLINIC CROSSING

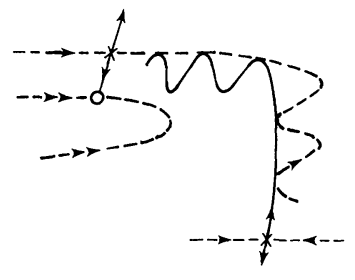

(5) HOMOCLINIC TANGENCY (1)

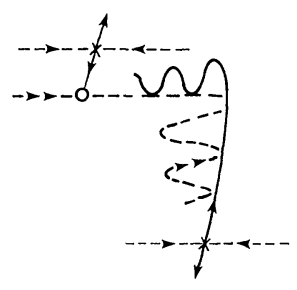

(2) HETEROCLINIC

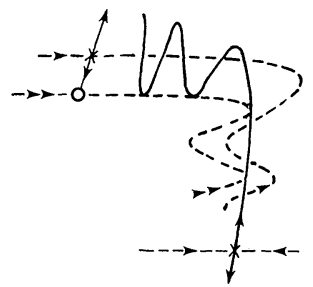

(4) HOMOCLINIC CROSSING HETEROCLINIC TANGENCY

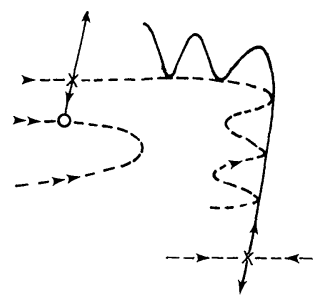

(6) HOMOCLINIC TANGENCY (II)

Fig. 9.4. Homo- and heteroclinic tangencies

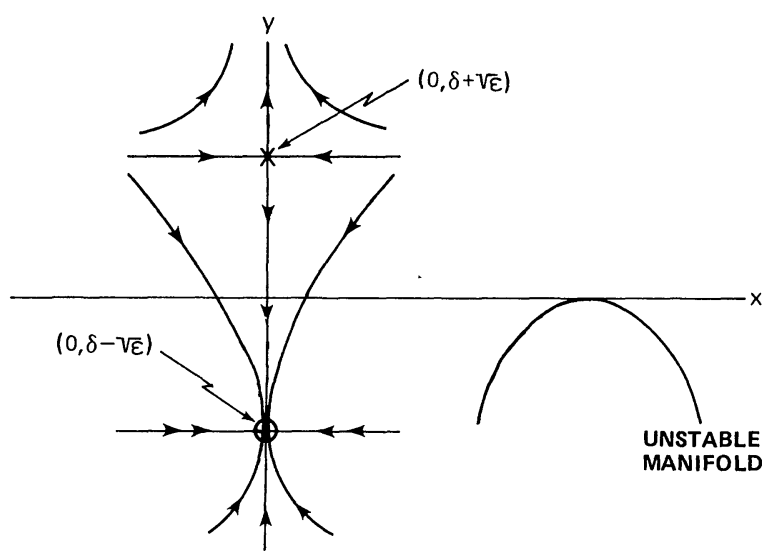

Fig. 9.5. Model based on Eq. (9.1) for the stable and unstable manifolds near the point $E$ in Fig. 9.1 


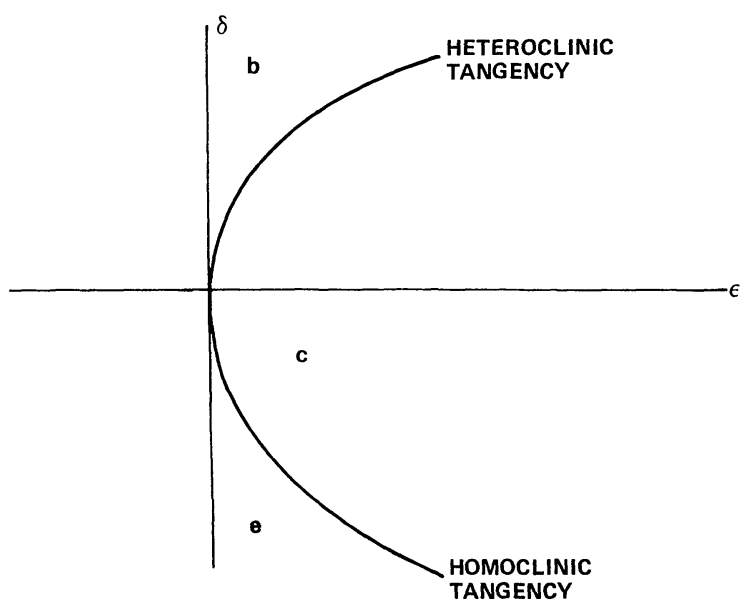

Fig. 9.6. Regions in the $(\delta, \varepsilon)$-plane corresponding to the behavior indicated in Fig. 8.3b, c, and e for the model (9.1)

boundary of the horn, with $\varepsilon$ positive inside the horn. Thus the curve $\delta=-\sqrt{\varepsilon}$ corresponds to the arc $E D$ in Fig. 9.1, while the curve $\delta=+\sqrt{\varepsilon}$ corresponds to the curve $E F$.

Along the arc $E F$, the invariant circle has the heteroclinic tangency shown in Fig. 9.4(1). Immediately below this arc, the unstable manifold of the saddle intersects transversely the strong stable manifold of the sink, as shown in Fig. 9.3c. Above this arc, the unstable manifold of the saddle comes into the sink tangent to the weak eigenspace, as shown in Fig. 9.3b.

The distinction between the structures of the invariant circle for parameter values above and below $E F$ is subtle but significant. As we know, the circle $A$ is a $C^{1}$ submanifold of the annular attractor block $B$ above the arc $J K$. Immediately below this arc, the circle fails to be $C^{1}$ because the tangent spaces to the sink oscillate wildly. However, the unstable manifold of the periodic saddle is compressed against the weak eigenspace of the sink, and hence this eigenspace is the tangent space to the invariant circle at the sink. If we say that the submanifold $A$ is a differentiable submanifold of $B$ if it has a tangent space at every point, then the region $E F K J E$ represents parameter values for which the invariant circle is a differentiable submanifold, but not a $C^{1}$ submanifold.

One should note here that the existence of the arc $E F$ implies the existence of the $\operatorname{arc} J K$. To be a little more precise, the existence of the point $E$ and the $\operatorname{arc} E F$ implies the existence of the point $J$ and the arc of homoclinic tangencies in $B \times P^{1}$ emanating from it. However, we are not certain about the termination of the arc from $J$, so that the point $K$ in Fig. 9.1 represents a guess. Indeed, it was our computer observations on the arc $E F$ which led us to the formulation of the loss of smoothness described in Sect. 8. We do not yet have the computer programs to follow the stable and unstable manifolds in $B \times P^{1}$, so our resolution of the arc $J K$ is based on computations in $B$ and is rather poor, particularly near the point $K$. 
As the parameters move down the resonance horn, it is possible that the unstable manifold of the saddle again becomes tangent to the strong stable manifold of the sink, but from a different orientation, as shown in Fig. 9.4(2) and (4). We found such behavior in the $1 / 8$ resonance horn, where it occurs for parameter values along the arc $I L$ in Fig. 9.1. Below this arc, there is no interaction between the unstable manifold and the strong stable manifold. However, both branches of the strong stable manifold pass directly to the outside of the annulus, as shown in Fig. 9.3d and f. Contrast this with region (a), where the two branches pass to opposite sides of the annulus. In region (d) the invariant circle has a cusp at the sink, as shown in Fig. 9.3d. In region (f) a homoclinic point has developed, and the attractor $A$ is no longer even topologically a circle.

At the point $I$, the unstable manifold of the saddle-node becomes tangent to its own strong stable manifold, as shown in Fig. 9.2(iv). This point is analogous to the point $E$ except again for the orientation. There is also another arc emerging from $I$ (labeled 6 in Fig. 9.1), so that the analogy with the point $E$ is complete. Along this arc, there is a homoclinic tangency, as shown in Fig. 9.4(6). Note again the different orientation, as contrasted with Fig. 9.4(5).

The existence of the point $I$ implies the existence of the quite remarkable region labeled $g$ in Fig. 9.1. In this region, there is no longer a homoclinic point; instead both branches of the stable manifold of the saddle come directly from the outside of the annulus, as shown in Fig. 9.3g. We find this region remarkable for two reasons :

(1) The attractor $A$ has broken into at least two components, one of which is the periodic sink with rotation number $1 / 8$. It appears that the complement of this sink has rotation numbers bounded away from $1 / 8$. For a given point $(a, b)$ in the region $g$, the set of rotation numbers assumed by points on the attractor $A$ for the map $F_{(a, b)}$ has a gap, and hence is not an interval. This property should be contrasted with the fact that the set of rotation numbers is always an interval for invertible or non-invertible maps of the circle.

(2) The development of the homoclinic tangency as the parameters approach the arc labeled 6 from inside of the region labeled $g$ seems to imitate in miniature the development of the homoclinic tangency discussed in Sect. 3 and shown in Fig. 3.3. This imitation leads us to speculate that the region $g$ itself may contain subregions where an analogous homoclinic tangency occurs for some higher order resonance. There may be an infinitely nested structure of such regions.

Just as remarkable as the existence of region $g$ is its termination further down the resonance horn. It terminates with the development of a homoclinic orbit near the point labeled $H$. The arc $H G$ is again an arc of heteroclinic tangencies, as shown in Fig. 9.4(4).

The arc $I H$ inside the resonance horn is probably an accumulation of left hand boundaries of other resonance horns analogous to the accumulation of right hand boundaries along $E D$ as shown in Fig. 7.2. We have not pursued this thought very far.

It is likely that a curve analogous to the curve $L I H G$ exists on the other side of the resonance horn. Also, there may be an arc of heteroclinic tangencies in $B \times P^{1}$ analogous to the arc $J K$ but occurring in region $d$. We have not as yet searched for either of these phenomena. 


\section{Other Phenomena Associated with the Periodic Sink}

We now turn our attention to the behavior of the map for parameter values inside the curve of double eigenvalues in the resonance horn. We have seen the behavior described below for every resonance horn that we have studied, but our observations are most complete for the $1 / 8$ resonance.

Immediately inside this curve of double eigenvalues, the eigenvalues at the sink are complex, and the invariant circle has the spirals shown in Fig. 9.3h. Further inside the horn the eigenvalues again become real but negative. Further still the sink loses stability by developing an eigenvalue of -1 and undergoing a perioddoubling bifurcation. The original $q$-periodic sink now has become a saddle with negative eigenvalues, and a new $2 q$-periodic sink has appeared.

A model of this period-doubling bifurcation for the $q^{\text {th }}$ iterate of the map near the sink is the following:

$$
(x, y) \rightarrow\left(-r x,-(1+\varepsilon) y+y^{3}\right),
$$

where $r$ is a fixed constant, $0<r<1$, and $\varepsilon$ is a small parameter. The origin is a fixed point of this map with eigenvalues $-r$ and $-(1+\varepsilon)$ for all values of $\varepsilon$. For $\varepsilon<0$, the origin is a sink with the $x$-axis as its strong stable manifold, as shown in Fig. 10.1a. Since the eigenvalues are negative, points approaching the sink oscillate back and forth across the origin. For $\varepsilon>0$, the origin is a saddle with the $x$-axis as its stable manifold and the $y$-axis as its unstable manifold. There is a periodic orbit of period 2 at $(x, y)=(0, \pm \sqrt{\varepsilon})$, as shown in Fig. $10.1 \mathrm{~b}$.

These events occur along curves in the parameter space, as sketched in Fig. 10.2. Assume that the eigenvalues of the sink are $\alpha$ and $\beta$. The boundary of the resonance horn is characterized by $\alpha=+1$. The first curve sketched inside the horn is where the two positive eigenvalues become equal, i.e., where $\alpha=\beta>0$. Between this curve and the next the eigenvalues are complex. The next curve is where the eigenvalues again become real, this time negative, i.e., where $\alpha=\beta<0$. The innermost curve is where $\alpha=-1$. Inside this curve, the original $q$-periodic sink has

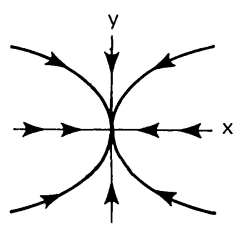

(a) $\epsilon<0$

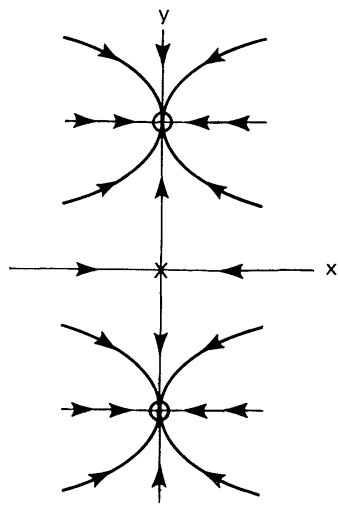

(b) $\epsilon>0$

Fig. 10.1a and b. A model based on (10.1) for period doubling bifurcation. a The eigenvalue of the sink $(0,0)$ in the $y$-direction is near -1 for $\varepsilon<0$. b A period two sink bifurcates from the origin as $\varepsilon$ increases from 0 leaving behind a saddle 


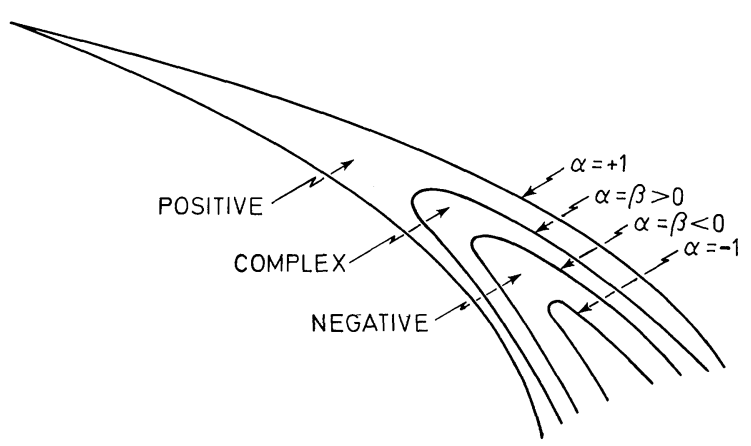

Fig. 10.2. Sketch of a typical resonance horn with regions where the eigenvalues $\alpha, \beta$ of the sink are positive, complex, and negative as indicated

become a saddle with negative eigenvalues, while the original $q$-periodic saddle has remained with positive eigenvalues. Immediately inside this last curve there is a $2 q$ periodic sink.

This story repeats itself infinitely often. The $2 q$-periodic sink develops complex, then negative, eigenvalues and undergoes a period doubling bifurcation, producing a $4 q$-periodic sink. The $4 q$-periodic sink itself loses stability, producing an $8 q$ periodic sink. This process apparently goes on forever, with all of the $2^{n} q$-periodic orbits having arisen for parameter values in some region further inside the horn. Other $k q$-periodic saddle-sink pairs arise spontaneously along other curves inside this region. For example, inside the $2 / 15$ resonance horn we found periodic sinks with period 45 and $75(k=3$ and $k=5)$.

The similarity of the bifurcations described above to the bifurcations of interval maps is striking. Although our computer evidence is rather sparse, none of it contradicts the conjecture that the sequence of successive bifurcations inside the resonance horn follows the same rules as the sequence for interval maps $[10,19$, 31].

However, one should be cautious about the analogy with interval maps. For example, the eigenvalue of a periodic sink for a map of the interval goes from positive values to negative values by passing through zero. Since zero eigenvalues are impossible for diffeomorphisms, this same transition must be accomplished by passing through complex eigenvalues. This difference may not be important, but one should also recall the region $g$ of Fig. 9.1. If there is some analogy between the bifurcation structures for diffeomorphisms of the plane and for maps of the interval, then one might also expect some analogy between these structures for diffeomorphisms of the annulus and for maps of the circle. However, we have already noted that the set of rotation numbers for the map $F_{(a, b)}$ with parameter values inside the region $g$ is not an interval. Since the set of rotation numbers for a map of the circle is always an interval, any analogy which exists may be somewhat weak.

Despite this caution, one is also struck by the similarity between an interval map and the visible attractor of $F_{(a, b)}$ for certain parameter values. For example, Fig. 10.3 shows the visible attractor for $(a, b) \approx(2.252,0)$. This parameter point is in 


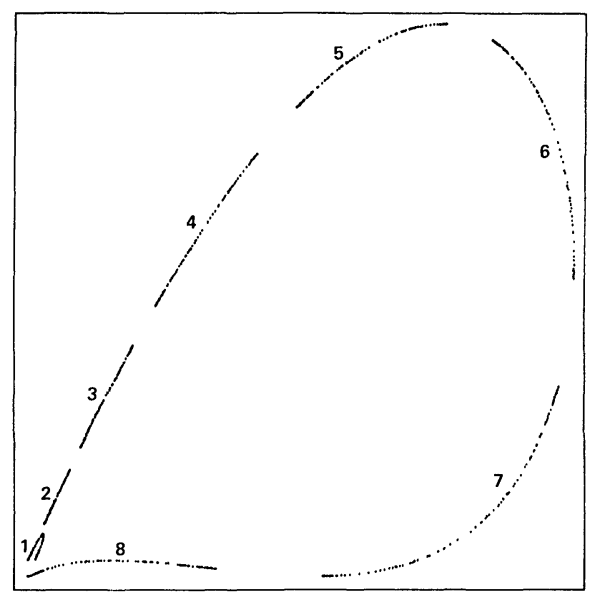

Fig. 10.3. Visible attractor for the delayed logistic map (3.2) with $a=2.2521185$. Note that this value of $a$ belongs to the segment $D D^{\prime}$ in Fig. 3.4, 7.2, and 9.1
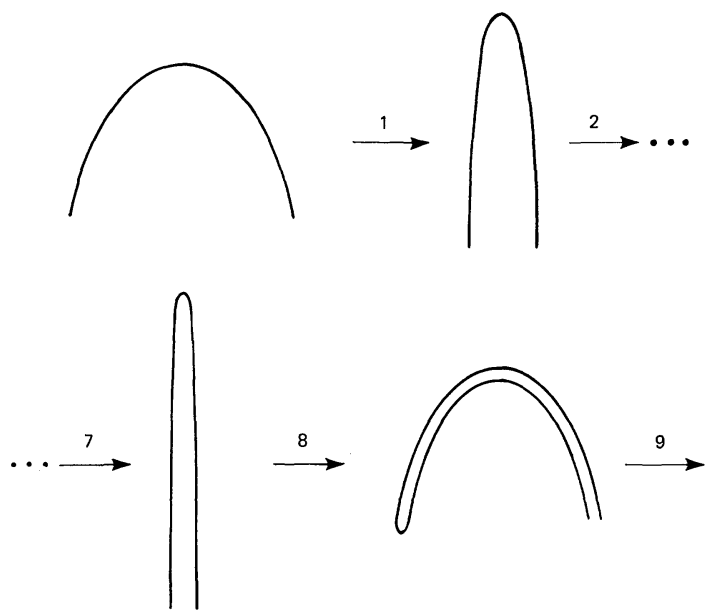

Fig. 10.4. Schematic representation of the action of eight iterations of the delayed logistic map (3.2) applied to the part of the attractor labeled 1 in Fig. 10.3

the $1 / 8$ resonance horn along the $a$-axis between points $D$ and $D^{\prime}$ in Fig. 9.1. There are no homoclinic points involving the $1 / 8$ saddle, and the rotation number of every point is $1 / 8$. Although the visible attractor appears at first glance to consist of eight arcs, a closer examination shows that these apparent arcs are extremely complicated sets. Suppose that the part of the attractor labeled 1 in Fig. 10.3 were an arc. The fate of that arc under eight iterations of the map is shown schematically in Fig. 10.4. In the first seven iterations the arc is compressed and stretched, while in the eighth iteration it is folded over on itself as shown. The overall effect on the original arc after eight iterations is similar to the effect on the unit interval of the map $x \rightarrow 4 x(1-x)$. Since the map $F_{(a, b)}$ is one-to-one, the 


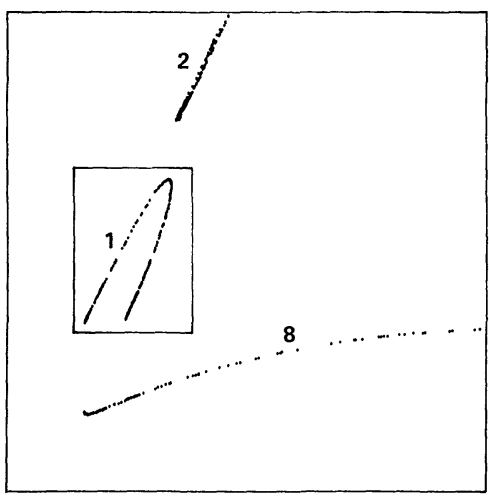

(a)

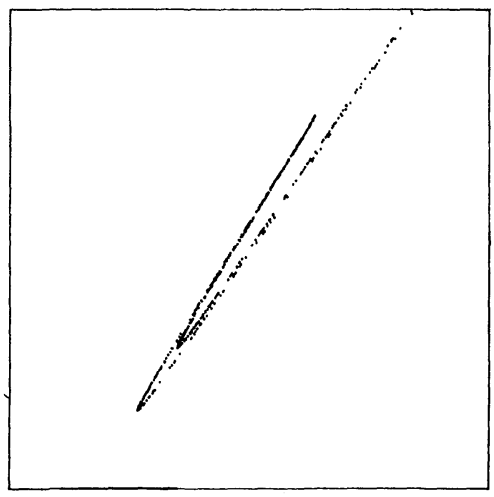

(c)

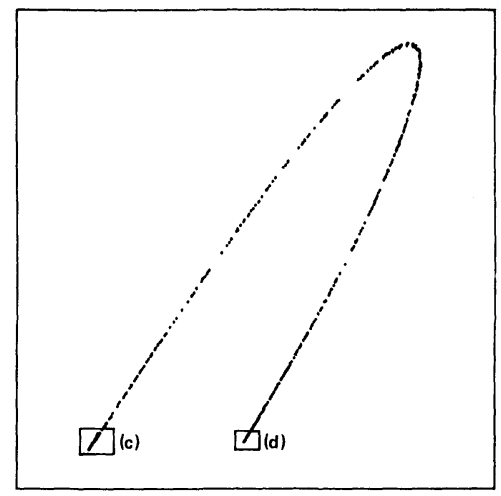

(b)

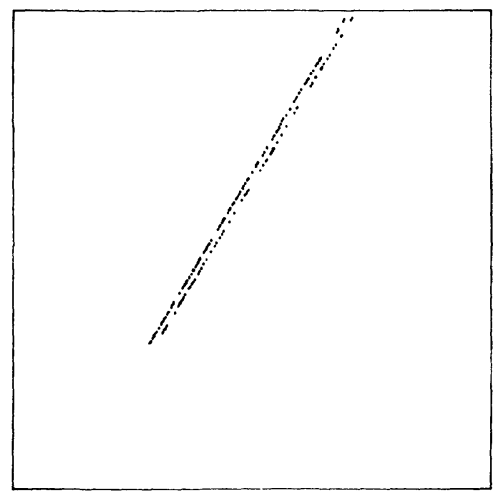

(d)

Fig. 10.5a-d. Enlargements of the part labeled 1 of the visible attractor shown in Fig. 10.3. Scales: b $x \in[0.018125,0.0575], y \in[0.0484375,0.10125] ; \mathbf{c} x \in[0.02350676,0.02556934], y \in[0.05236221$, $0.05519533] ; \mathbf{d} x \in[0.03652778,0.03750160], y \in[0.053174,0.05445258]$

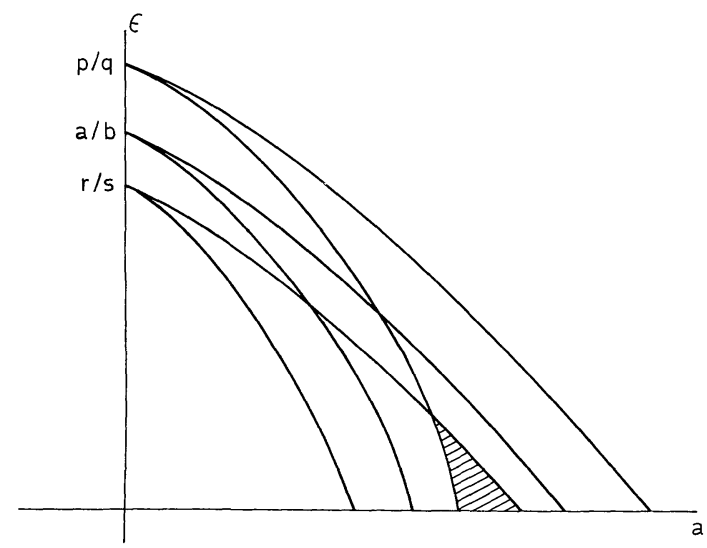

Fig. 10.6. Sketch of resonance horns for rotation numbers $p / q, a / b$, and $r / s$ with $r / s<a / b<p / q$. The shaded region is the intersection of the $r / s$ and $p / q$ horns and is contained completely in $a / b$ horn 
original segment could not possibly be an arc. A sequence of enlargements of this segment is shown in Fig. 10.5. Again, note the similarity of these pictures with those obtained by Hénon [13].

To end this discussion of phenomena associated with the sink, we wish to speculate on the relation between overlapping horns and the complicated sequence of bifurcations described above. To be more specific, suppose that two resonance horns $p / q$ and $r / s$ cross as shown in Fig. 10.6. Consider the $a / b$ resonance horn, where $a / b$ is some rational number between $p / q$ and $r / s$. Now concentrate on parameter values in the region of overlap between the $p / q$ horn and the $r / s$ horn. This region lies inside the $a / b$ resonance horn. Using the constructions of Sect. 7, we can find infinitely many different periodic orbits with rotation number $a / b$. In particular, we can find $2^{n} b$-periodic and $k b$-periodic orbits for all positive $n$ and $k$. It is tempting to conjecture that these orbits have all arisen from bifurcations related to the original $a / b \operatorname{sink}$ in some analogy with similar orbits for an interval map.

\section{One Parameter Families}

Many important systems have naturally only one parameter, or at least are studied by varying only one parameter. However, as we have seen in this paper, the phenomena associated with bifurcations from an invariant circle are more easily understood in two parameter families. With this increased understanding, it is easier to see patterns in the apparent complexity of one parameter families.

We conclude our report with a return to the original model of delayed regulation, as an example of what we believe to be typical behavior for one parameter families. This model is given by the family $F_{a}$ of Sect. 3 . Recall that $F_{a}$ is the one parameter subfamily obtained by letting $b=0$ in the family $F_{(a, b)}$ of Sect. 5 .

We studied the behavior of $F_{a}$ as $a$ varies from 2 to $a^{*} \approx 2.2701$. Recall that there is a Hopf bifurcation at $a=2$ from a stable fixed point to a smooth attracting invariant circle. This circle transforms into a strange attractor as $a$ increases to $a^{*}$. Our original naive hypothesis was that the invariant circle would lose smoothness, gradually and monotonically, as $a$ increased. Our goal was to pin down the precise value of $a$ for which the attractor made the transition from a circle to a strange attractor. Of course, we now know that the attractor changes back and forth from a circle to a non-circle many times as $a$ increases from 2 to $a^{*}$.

As $a$ varies from 2 to 2.17, simple computer simulations show a visible attractor that appears to be a circle conjugate to a rigid rotation. We know from the general theory, however, that there should be a resonance at every rational rotation number [1]. These resonances correspond to the intersection of the $a$-axis with the various resonance horns between $1 / 6$ and $1 / 7$. When we used a numerical Newton's method to find periodic orbits, we easily found these resonances in the expected locations. However, the interval of $a$ for which a particular resonance occurs is far too small to be seen at the scale of Fig. 3.4.

One should note how the smoothness of the invariant circle changes as $a$ moves across this infinite number of resonance horns. The circle is $C^{\infty}$ on the boundary of each horn, while inside it is only $C^{r}$ for some $r<\infty$. Thus the smoothness of the 
circle as a function of the parameter is far from monotone, changing infinitely often from $C^{\infty}$ to $C^{r}$.

For $a$ in the range between approximately 2.177 and 2.200 , a $1 / 7$ resonance occurs, and the visible attractor shows only a period 7 sink. However, the invisible attractor develops a spiral on part of the interior of this range. Thus the circle loses all of its smoothness during this resonance, becoming a non-differentiable submanifold. Although our computer studies were not conclusive, our best guess is that the circle regains all its smoothness, becoming once again $C^{\infty}$ near $a=2.200$.

As $a$ varies from 2.20 to 2.22 the visible attractor takes on many resonances, which become easier and easier to find as $a$ increases. The simple computer simulations show only what appear to be stable periodic orbits for some values of $a$ interspersed with quasi-periodic orbits for other values. However, for $a$ in this range, we are passing through those parts of the resonance horns which exhibit the structures discussed in Sects. 9 and 10, and the behavior of the invisible attractor $A$ is extremely complicated. Numerical computations on the unstable manifold show many homoclinic and heteroclinic orbits arising and disappearing through tangencies.

One should note here that the existence of a homoclinic orbit, and hence of the infinite number of complicated periodic orbits implied by it, does not necessarily show up in simple computer simulations. All of the orbits constructed in Sect. 7 are unstable and may not be part of the visible attractor. In fact, for parameter values in the regions marked $e$ and $f$ in Fig. 9.1, one usually sees in the visible attractor only the periodic sink associated with the resonance horn. Thus the complicated behavior associated with homoclinic orbits occurs behind the scenes, as part of the transient behavior of the system. It appears that it is only when the homoclinic structures interact with the structures of Sect. 10 that they become part of the visible attractor.

As we increase the parameter above 2.22 we encounter a $2 / 15$ resonance from approximately 2.2218 to 2.2344. As a varies across this interval, the visible attractor begins as a period 15 sink, appears to undergo all of the bifurcations discussed in Sect. 10, appears then to undergo all of these bifurcations in reverse order, and ends up again as a period $15 \operatorname{sink}$. Moreover, for $a$ in part of the interior of this range, the $15^{\text {th }}$. iterate of the map seems to have a miniature strange attractor, similar to that shown in Fig. 10.5. This miniature attractor is quite small, however, and can be missed without careful scrutiny.

If one returns with this more careful scrutiny to parameter values below 2.22, one finds other instances where some of the bifurcations of Sect. 10 occur. For example, the $3 / 22$ resonance undergoes many bifurcations, becoming quite complicated in the interior of its range. Another example is the $4 / 29$ resonance, where the period 29 sink bifurcates to a period 58 sink.

At the end of the $2 / 15$ resonance we find that the visible attractor suddenly jumps from a period 15 sink to a period 8 sink when $a$ passes 2.2344 . In fact, at this value of $a$ we find these two sinks coexisting. Simple computer simulations show one sink or the other, depending on the initial point. The coexistence of stable periodic orbits with different rotation numbers has been discussed before $[8,16]$.

During the $1 / 8$ resonance, the complexity of the visible attractor seems to be essentially monotonically increasing. We start with a period 8 sink which 
undergoes all the doubling and other bifurcations, eventually arriving at the structure shown in Figs. 10.3-10.5. It continues to grow in complexity, as a homoclinic point develops and begins to interact with the already complicated structure. The $1 / 8$ resonance region continues all the way to $a^{*}$ (and beyond). At the beginning of the $1 / 8$ resonance, the invisible attractor already has a homoclinic orbit. This orbit disappears in a homoclinic tangency at $a \approx 2.2414$, but by this time the visible attractor has undergone two period-doubling bifurcations. There is an interval from $a \approx 2.2414$ to $a \approx 2.2520$ where the only rotation number present in $1 / 8$, even though the visible attractor is quite complicated.

We believe that the above sequence of events is typical in outline for one parameter families of diffeomorphisms of the plane. Our analysis makes it clear that this behavior is best understood by the study of one parameter subfamilies of two parameter families. One can see that it is quite difficult to draw a one parameter subfamily through the infinite number of resonance horns without passing many times through regions of relative simplicity interspersed with regions of relative complexity. In one parameter families the transition from simple to complicated behavior is itself quite complicated.

Acknowledgements. During the research on this paper the authors benefitted from conversations with many individuals, too numerous to name. However, we wish to give particular thanks to Clarence Lehman for his efforts during the early stages of this work. Many of the original insights and programming techniques came from our association with him.

D. G. Aronson was partially supported by NSF Grant MSC 78-02158, R. P. McGehee was partially supported by NSF Grant MSC 79-01998, and G. R. Hall was supported by a grant-in-aid of research from the Graduate School of the University of Minnesota. The computations were done in the Dynamical Systems Laboratory in the University of Minnesota School of Mathematics. The Laboratory is supported by NSF Grant 78-02173, a grant-in-aid of research from the Graduate School of the University of Minnesota, and a grant from the School of Mathematics of the University of Minnesota.

\section{References}

1. Arnol'd, V.I.: Loss of stability of self-oscillations close to resonance and versal deformations of equivarient vector fields. Funct. Anal. Appl. 11, 1-10 (1977)

2. Aronson, D.G., Chory, M.A., Hall, G.R., McGehee, R.P.: A discrete dynamical system with subtly wild behavior. New approaches to nonlinear problems in dynamics, Holmes, P. (ed.). SIAM 1980

3. Bowen, R.: On axiom $A$ diffeomorphisms. CBMS Regional Conference Series in Mathematics, No. 35. Providence, Rhode Island: Am. Math. Soc. 1978

4. Conley, C.: Isolated invariant sets and the Morse index. CBMS Regional Conference Series in Mathematics, No. 38. Providence, Rhode Island: Am. Math. Soc. 1978

5. Conley, C.: Hyperbolic sets and shift automorphisms. Dynamical systems: theory and applications. In: Lecture Notes in Physics, Vol. 38, Moser, J. (ed.), pp. 539-549. Berlin, Heidelberg, New York: Springer 1975

6. Curry, J., Yorke, J.: A transition from Hopf bifurcation to chaos: computer experiments on maps in $R^{2}$. The structure of attractors in dynamical systems. In: Lecture Notes in Mathematics, Vol. 668, pp. 48-68. Berlin, Heidelberg, New York: Springer 1978

7. Fenichel, N. : Persistence and smoothness of invariant manifolds for flows, Ind. Univ. Math. J. 21, 193-226 (1971)

8. Flaherty, J., Hoppensteadt, F.: Frequency entrainment of a forced van der Pol oscillator. Stud. Appl. Math. 58, 5-15 (1978)

9. Guckenheimer, J. : A strange, strange attractor. The Hopf bifurcation theorem and its applications, Marsden, J., McCracken, M. (eds.), pp. 368-381. Berlin, Heidelberg, New York: Springer 1976

10. Guckenheimer, J.: On the bifurcation of maps of the interval. Invent. Math. 39, 165-178 (1977) 
11. Guckenheimer, J., Williams, R.F.: Structural stability of Lorenz attractors. Publ. Math. I.H.E.S. 50, 307-320 (1979)

12. Hartman, P.: Ordinary differential equations. New York: Wiley 1964

13. Hénon, M.: A two-dimensional mapping with a strange attractor. Commun. Math. Phys. 50, 67-77 (1976)

14. Iooss, G.: Topics in bifurcation of maps and applications. Math. Stud. 36. Amsterdam: NorthHolland 1979

15. Levi, M.: Qualitative analysis of the periodically forced relaxation oscillators. Mem. AMS 244, 1981

16. Levinson, N.: A second order differential equation with singular solutions. Ann. Math. 50, 127-153 (1949)

17. Lorenz, E.N.: Deterministic nonperiodic flow. J. Atmos. Sci. 20, 130-141 (1963)

18. Maynard Smith, J.: Mathematical ideas in biology. Cambridge: Cambridge University Press 1971

19. Milnor, J., Thurston, W.: On iterated maps of the interval, I, II (preprint)

20. Newhouse, S.: Diffeomorphisms with infinitely many sinks. Topology 13, 9-18 (1974)

21. Poincaré, H.: Les méthodes nouvelles de la mécanique céleste. Vol. 3. Paris: Gauthiers-Villars 1899

22. Pounder, J.R., Rogers, T.D.: The geometry of chaos: dynamics of a nonlinear second-order difference equation. Bull. Math. Biol. 42, 551-597 (1980)

23. Rademacher, H.: Lectures on elementary number theory. New York: Blaisdell 1964

24. Ruelle, D.: A measure associated with axiom $A$ attractors. Am. J. Math. 98, 19-64 (1976)

25. Ruelle, D.: Strange attractors. The Mathematical Intelligencer 2, 126-137 (1980)

26. Ruelle, D., Takens, F.: On the nature of turbulence. Commun. Math. Phys. 20, 167-192 (1971)

27. Smale, S.: Diffeomorphisms with many periodic points. Differential and combinatorial topology, pp. 63-80. Princeton, N.J.: Princeton University Press 1965

28. Stein, P.R., Ulam, S.M.: Nonlinear transformation studies on electronic computers. Rozprawy Matem. 39, 3-65 (1964)

29. Takens, F.: Forced oscillations and bifurcations. Applications of global analysis. Commun. Math. Inst. Rikjsuniversitat Utrecht

30. Williams, R.F.: Expanding attractors. Publ. Math. I.H.E.S. 43, 169-203 (1974); see also Proceedings of the Mount Aigual Conference on Differential Topology, Univ. of Montepellier, 1969

31. Zharkovsky, A.N.: Coexistence of cycles of a continuous map of a line into itself. Ukrain. Mat. Z. 16, 61-71 (1974)

Communicated by D. Ruelle

Received May 8, 1981 